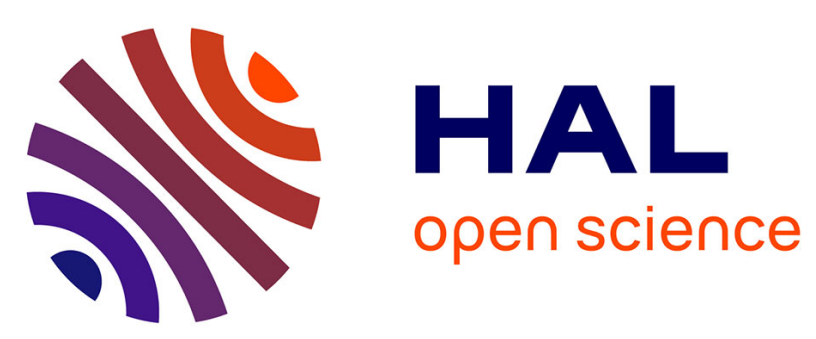

\title{
Analysis of the ECGI inverse problem solution with respect to the measurement boundary size and the distribution of noise
}

Mohammed Addouche, Nadra Bouarroudj, Fadhel Jday, Jacques Henry, Nejib Zemzemi

\section{To cite this version:}

Mohammed Addouche, Nadra Bouarroudj, Fadhel Jday, Jacques Henry, Nejib Zemzemi. Analysis of the ECGI inverse problem solution with respect to the measurement boundary size and the distribution of noise. Mathematical Modelling of Natural Phenomena, 2019, 10.1051/mmnp/2018061 . hal01923800

\section{HAL Id: hal-01923800 \\ https://hal.inria.fr/hal-01923800}

Submitted on 15 Nov 2018

HAL is a multi-disciplinary open access archive for the deposit and dissemination of scientific research documents, whether they are published or not. The documents may come from teaching and research institutions in France or abroad, or from public or private research centers.
L'archive ouverte pluridisciplinaire HAL, est destinée au dépôt et à la diffusion de documents scientifiques de niveau recherche, publiés ou non, émanant des établissements d'enseignement et de recherche français ou étrangers, des laboratoires publics ou privés. 


\title{
ANALYSIS OF THE ECGI INVERSE PROBLEM SOLUTION WITH RESPECT TO THE MEASUREMENT BOUNDARY SIZE AND THE DISTRIBUTION OF NOISE
}

\author{
Mohammed Addouche ${ }^{1}$, Nadra Bouarroudj ${ }^{2}$, Fadhel Jday ${ }^{3}$, Jacques Henry ${ }^{4,5}$ \\ AND NEJIB ZEMZEMI ${ }^{4,5}$
}

\begin{abstract}
In this work, we analyze the influence of adding a body surface missing data on the solution of the electrocardiographic imaging inverse problem. The difficulty comes from the fact that the measured Cauchy data is provided only on a part of the body surface and thus a missing data boundary is adjacent to a measured boundary. In order to construct the electrical potential on the heart surface, we use an optimal control approach where the unknown potential at the external boundary is also part of the control variables. We theoretically compare this case to the case where the Dirichlet boundary condition is given on the full accessible surface. We then compare both cases and based on the distribution of noise in the measurements, we conclude whether or not it is worth to use all the data. We use the method of factorization of elliptic boundary value problems combined with the finite element method. We illustrate the theoretical results by some numerical simulations in a cylindrical domain. We numerically study the effect of the size of the missing data zone on the accuracy of the inverse solution.
\end{abstract}

Mathematics Subject Classification. 12A34, 56B78 .

November 15, 2018.

\section{INTRODUCTION}

Electrocardiographic imaging (ECGI) is a new technology that allows to non-invasively reconstruct the electrical activity of the heart from measurements on the body surface and geometrical information of the torso. This clinical tool is used by cardiologists in order to localize arrhythmogenic substrates, such as atrial and ventricular fibrillations. The current clinical tool consists of a vest containing 252 electrodes that are used to measure the electrical potential at the body surface and software used to calculate the electrical potential on the heart surface. These electrodes are distributed on the body surface. The mathematical method used behind the tool is the Method of Fundamental Solutions (MFS) presented in the work by Wang and Rudy [25]. However, the 252 electrodes are not equally distributed on the torso surface and some regions are more covered than

Keywords. Inverse problem; electrocardiography imaging; Factorization method; Riccati equations.

1 Abou Bekr Belkaid University, Tlemcen, Algeria. e-mail: addouchelmd@gmail.com

2 Department of Mathematics and informatics, ENP, Oran,Maurice Audin, Algeria. e-mail: nadra.belaib@yahoo.fr

3 Umm al-Qura University, Mekkah KSA. e-mail: fadheldj@yahoo.com

${ }^{4}$ INRIA, Bordeaux Sud-Ouest, 200 Avenue de la vielle Tour 33405 Talence Cedex France. e-mail: Jacques Henry \& nejib.zemzemi@inria.fr

5 IHU LIRYC, Electrophysiology and Heart Modeling Institute, Pessac, France. 
others. Moreover, in clinical practice some electrodes are not considered in the inverse problem. Sometimes because they are not in contact with the patient's skin and sometimes because of the high noise registered on some electrodes. This means that there are regions where we know the potential and others where we don't have any information. But in both cases, we know that we have zero flux boundary condition on the whole body surface. In different studies, for instance John et al. [11], Ghodrati et al. [13] and Oostendorp et al. [23], authors reconstruct these missing BSPs by interpolating them from the well-measured signals: In this case Oostendorp et al. [23] used a Laplacian interpolation of the electrical potential on the body surface. Burnes et al. [11] presented a forward-inverse method, where they solved the inverse problem on an intermediate surface between the torso and the heart surfaces using the recorded potential by the electrodes. Then they solved the forward problem to recover the potential on the whole body surface. Once they have the value of the potential on the whole body surface, they solved the ECGI inverse problem to obtain the potential on the heart surface. Ghodrati et al. [13] used a Bayesian approach to interpolate the electrical PSPs before solving the inverse problem. In a recent work, Bear et al. [8] showed that in some cases the interpolation does not improve the inverse solution and sometimes it even worsens the quality of the reconstructed potentials. In almost of the cited works as well as most of reported in the literature for solving the ECGI inverse problem, the mathematical problem was formulated using a transfer matrix approach. This approach has been first introduced by Roger et al. [7]. The transfer matrix was later computed using different approaches like boundary elements, finite elements or MFS. Recent works (Bouyssier et al. [10]; Hariga et al. [15]; Zemzemi, [26]; Zemzemi et al. [27] ) presented novel approaches based on recent advances in boundary-value inverse problem techniques. These works use an energy-based cost function. The theoretical study of these methods has been reported by Ben Abda et al. [1], Andrieux et al. [5] and Azaiez et al. [6]. In this work we use the method of boundary value factorization introduced for solving data completion by Ben Abda et al. [1], which has already been used for solving the ECGI problem in the work by Bouyssier et al. [10]. Our goal in this work is to introduce a new mathematical formulation that takes into account the missing data on the body surface without using interpolation methods. The missing BSPs would be part of the control problem. However, applied to the ECGI inverse problem, the methodology developed in this paper may be applied to any data completion problem for the Laplace equation with missing data on the accessible boundary.

\section{Continuous Problem and Factorization Method}

For the sake of simplicity we study a model problem where the considered domain is a cylinder. The cylindrical geometry and the Laplace operator make the presentation of the factorization method easier. The method can be generalized to regular non-cylindrical domains and to more general self-adjoint second-order elliptic operators. The generalization for a three dimensional realistic domain can be done and will be presented in a further article.

Let's consider the cylindrical domain $\Omega$ is a cylinder in $\left.\mathbb{R}^{n}, \Omega=\right] 0, a[\times \mathcal{O}$, where $\mathcal{O}$ is a bounded domain in $\mathbb{R}^{n-1}$. The length of the cylinder $a$ is a strictly positive real number. We denote by $\partial \Omega$ the boundary of the cylinder: $\partial \Omega=\Gamma_{0} \cup \Gamma_{a} \cup \Sigma$, where $\left.\Sigma=\right] 0, a\left[\times \partial \mathcal{O}\right.$ is the lateral boundary of $\Omega, \Gamma_{0}=\Gamma_{0}^{m} \cup \Gamma_{0}^{u}=\{0\} \times \mathcal{O}$ and $\Gamma_{a}=\{a\} \times \mathcal{O}$ are the faces. The section $\mathcal{O}$ could be partitioned as $\mathcal{O}=\mathcal{O}^{m} \cup \mathcal{O}^{u}$, so that $\{0\} \times \mathcal{O}^{m}=\Gamma_{0}^{m}$ and $\{0\} \times \mathcal{O}^{u}=\Gamma_{0}^{u}$. We suppose that $\Gamma_{0}$ is an accessible boundary, which means that we can construct the electrical potential. On the other hand $\Gamma_{a}$ is an inaccessible boundary, which means that we can't construct any measurements on it. For the reasons mentioned previously, the boundary $\Gamma_{0}^{m}$ is the part of $\Gamma_{0}$ where we have measurements of the potentials and in $\Gamma_{0}^{u}$ we don't have electrical potential measurements. We denote by $x \in \mathbb{R}$ the coordinate along the axis of the cylinder and by $y \in \mathbb{R}^{n-1}$ the coordinates in the section, perpendicular to the axis. The schematic representation of the domain $\Omega$ and all its boundaries is represented in Figure (1). 


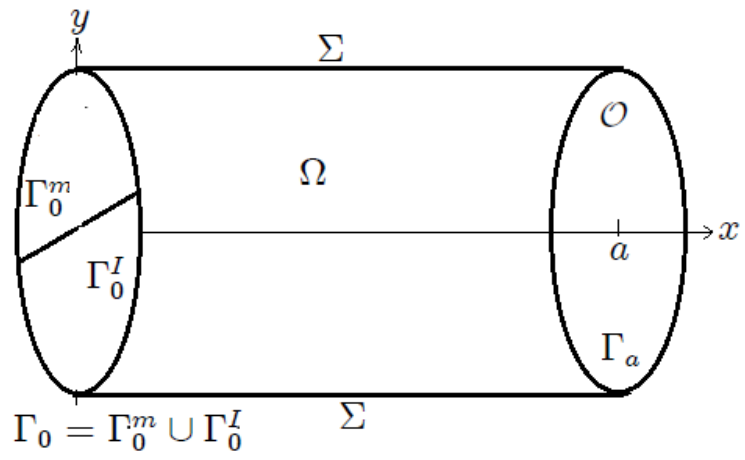

FiguRE 1. Schematic representation of the cylindrical domain used in the study.

\subsection{Statement of the Problem}

In this paper, we consider the following problem on $\Omega$ with Cauchy data on $\Gamma_{0}^{m}$ :

$$
\left(\mathcal{P}_{0}\right)\left\{\begin{array}{ccc}
-\Delta u=0 & \text { in } & \Omega, \\
u=0 & \text { on } & \Sigma, \\
\frac{\partial u}{\partial n}=0 & \text { on } & \Gamma_{0}, \\
u=T & \text { on } & \Gamma_{0}^{m} .
\end{array}\right.
$$

We recall the Sobolev space $H_{00}^{\frac{1}{2}}(\mathcal{O})$ defined in Theorem 11.7, p.72 of [21] as the $\frac{1}{2}$ interpolate between $H_{0}^{1}(\mathcal{O})$ and $L^{2}(\mathcal{O})$ and we denote by $\left(H_{00}^{\frac{1}{2}}(\mathcal{O})\right)^{\prime}$ its dual space. The boundary $\Gamma_{0}^{m}$ is the measured portion of the surface of $\Gamma_{0}$ i.e the part of $\Gamma_{0}$ where we have both Dirichlet $(u=T)$ and Neumann $\left(\frac{\partial u}{\partial n}=0\right)$ boundary conditions. The boundary $\Gamma_{0}^{u}$ is the unmeasured part of the surface $\Gamma_{0}$ i.e. it is the part of $\Gamma_{0}$ where we only know that we have a Neumann boundary condition $\left(\frac{\partial u}{\partial n}=0\right)$, whereas no boundary condition is available on $\Gamma_{a}$. The aim of this paper is to recover these missing boundary data exploiting the over-specified data on $\Gamma_{0}^{m}$. The existence of a solution of this problem is not insured for arbitrary Cauchy data. This problem is treated in [5], [12], [2] in a general domain $\Omega$ having a boundary: $\partial \Omega=\Gamma_{0} \cup \Gamma_{a}$. In [5] the missing boundary data recovery problem is rephrased as a control problem with two states, each one satisfying one boundary condition on $\Gamma_{0}^{m}$, the control being the unknown boundary conditions on $\Gamma_{a}$. The numerical methods proposed in [3-5] are iterative and therefore, for each new data, they require the resolution of many intermediate forward problem to solve the data completion problem . In the electrocardiography application, one should solve the Cauchy problem at many time steps in order to recover the dynamics of the electrical wave on the heart. For certain pathologies, one should solve the Cauchy problem hundreds or thousands of times in order to obtain the dynamics of the electrical information on the heart surface. In terms of computational cost, this reduces the competitiveness of the iterative methods like in [5], [15], [2], [20] and [26]) and gives more advantage for mesh-free methods like MFS ( [25]), where a transfer matrix is computed once for all. In this work we use the same energy function as defined in [5], but we formulate it at the continuous level using Dirichlet-Neumann and Neumann-Dirichlet mappings. These operators are time-independent; they only depend on the geometry. After computing these operators, we compute the inverse solution by solving a linear problem on $\Gamma_{a}$. We make use of the factorization method which transforms the elliptic boundary value problem into two parabolic ones. This approach allows to compute with a good accuracy the Dirichlet-Neumann and Neumann-Dirichlet mappings on a sequence of 
surfaces flowing from $\Gamma_{0}$ to $\Gamma_{a}$. It allows the direct evaluation of the missing boundary data for any new Cauchy data without re-computation of the operators.

\section{Formulation of the data COMPLETION PROBlem as AN OPtimal CONTROL PRoblem}

We assume we know exactly the observation $T$ on $\Gamma_{0}^{m}$ corresponding to the boundary value problem and we seek to determine the Dirichlet and Neumann boundary conditions on $\Gamma_{a}$ and $\Gamma_{0}^{u}$ supplementing the problem $\left(\mathcal{P}_{0}\right)$, i.e. the data of the electric potential $\tau$ and its derivative $\eta$ on the surface $\Gamma_{a}$ and the potential $\tau^{\prime}$ on $\Gamma_{0}^{u}$ such that the overdetermined boundary value problem $\left(\mathcal{P}_{0}\right)$ given by:

$$
\left\{\begin{array}{ccc}
-\Delta u=0 & \text { in } & \Omega, \\
u=0 & \text { on } & \Sigma, \\
\frac{\partial u}{\partial n}=0 & \text { on } & \Gamma_{0}, \\
u=T & \text { on } & \Gamma_{0}^{m}, \\
u=\tau^{\prime} & \text { on } & \Gamma_{0}^{u}, \\
u=\tau, \frac{\partial u}{\partial n}=\eta & \text { on } & \Gamma_{a},
\end{array}\right.
$$

has a solution. By Holmgren's theorem such conditions are unique and we denote them respectively by $t, \varphi$, and $\hat{\tau^{\prime}}$. In the real life inverse cardiac imaging application, the torso surface $\Gamma_{0}$ has it self no boundary. In the simplified cylindrical case, in order to avoid unnecessary technicalities, we make the following assumption $(\mathcal{H})$ on the subset $\Gamma_{0}^{m}$ and $\Gamma_{0}^{u}$ of $\Gamma_{0}$ :

$$
(\mathcal{H})\left\{\begin{array}{c}
\text { either } \partial \Gamma_{0} \subset \partial \Gamma_{0}^{m} \\
\text { or } \partial \Gamma_{0} \cap \partial \Gamma_{0}^{m}=\varnothing
\end{array}\right.
$$

To deal with well posed problems we have to make precise the spaces where $T$ is given and $\tau^{\prime}$ is sought. For that purpose we need the following lemma.

Lemma 3.1. Consider the open bounded set $\Omega \subset \mathbb{R}^{n}$ with regular boundary $\Gamma$. Assume $\Omega$ is separated into two open parts $\Omega_{0}$ and $\Omega_{1}$ with a common boundary $\gamma_{0}^{1}: \Omega=\Omega_{0} \cup \gamma_{0}^{1} \cup \Omega_{1}$, satisfying the assumption $(\mathcal{H})$. Let $u \in H_{00}^{\frac{1}{2}}(\Omega)$ such that $u \equiv 0$ in $\Omega_{1}$. Then $\left.u\right|_{\Omega_{0}} \in H_{00}^{\frac{1}{2}}\left(\Omega_{0}\right)$.

Proof 3.1. The proof is inspired from that of Theorem 11.7 in [21]. By partition of unity and the use of local maps, the problem is reduced to the simplified geometry: $\Omega=\mathbb{R}^{n}, \Gamma=\left\{x_{n}=0\right\}, \Omega_{0}=\left\{x_{n}<0\right\}, \Omega_{1}=\left\{x_{n}>0\right\}$. From Theorem 10.2 p 52 of [21], since $u \in H^{\frac{1}{2}}\left(\mathbb{R}^{n}\right)$, we have :

$$
\left(\int_{\mathbb{R}^{n}}|u|^{2} d x+\sum_{i=1}^{n} \int_{0}^{\infty} \mu^{-2} \int_{\mathbb{R}^{n}}\left|u\left(x_{1}, . ., x_{i}+\mu, \ldots, x_{n}\right)-u(x)\right|^{2} d x d \mu\right)^{\frac{1}{2}}<\infty .
$$

Therefore, setting $x=\left(x^{\prime}, x_{n}\right)$, in particular for $i=n$ we have:

$$
\left(\int_{0}^{\infty} \mu^{-2} \int_{\mathbb{R}^{n}}\left|u\left(x^{\prime}, x_{n}+\mu\right)-u(x)\right|^{2} d x d \mu\right)^{\frac{1}{2}}<\infty .
$$

But since by assumption $u(x) \equiv 0$ in $x_{n}<0$, and as $u \in H^{\frac{1}{2}}\left(\mathbb{R}_{+}^{n}\right)$, where $R_{+}^{n}=\left\{x_{n}>0\right\}$, we have:

$$
\left(\int_{0}^{\infty} \mu^{-2} \int_{\mathbb{R}_{+}^{n}}\left|u\left(x^{\prime}, x_{n}+\mu\right)-u(x)\right|^{2} d x d \mu\right)^{\frac{1}{2}}<\infty .
$$


From the two previous estimation, we deduce

$$
\left(\int_{0}^{\infty} \mu^{-2} d \mu \int_{\mathbb{R}^{n-1}} d x^{\prime} \int_{0}^{\mu}\left|u\left(x^{\prime}, x_{n}\right)\right|^{2} d x d \mu\right)^{\frac{1}{2}}<\infty .
$$

Integrating by parts in $t$ we have:

$$
\left(\int_{\mathbb{R}_{+}^{n}} \frac{1}{x_{n}}|u(x)|^{2} d x\right)^{\frac{1}{2}}<\infty .
$$

This is the regularity condition in the neighbourhood of $\Gamma$ for $u$ to belong to $H_{00}^{\frac{1}{2}}\left(\Omega_{1}\right)$ (Theorem 11.7 $p 66$ in [21]).

Thanks to assumption $(\mathcal{H})$, it is possible to construct a extension $\widetilde{T}$ of $T$ in $\Gamma_{0}^{u}$ such that:

$$
T_{0}=\left\{\begin{array}{c}
T \text { on } \Gamma_{0}^{m} \\
\widetilde{T} \text { on } \Gamma_{0}^{u}
\end{array} \quad T_{0} \in H_{00}^{\frac{1}{2}}\left(\Gamma_{0}\right) .\right.
$$

Let $\gamma_{m}^{u}$ be the common boundary to $\Gamma_{0}^{m}$ and $\Gamma_{0}^{u}$. By $(\mathcal{H}), \gamma_{m}^{u}$ is included in an open subset relatively compact in $\Gamma_{0}$. Then the extension can be made locally by symmetry with respect to $\gamma_{m}^{u}$ ( $\operatorname{cf}[21]$ ). The functions $\widetilde{T}$ and $\tau^{\prime}$ have to satisfy some compatibility conditions with $T$ on $\gamma_{m}^{u}$ in order to satisfy the restrictions on $\Gamma_{0}^{u}$ of functions of $H_{00}^{\frac{1}{2}}\left(\Gamma_{0}\right)$ To avoid this problem we add a bias to $\tau^{\prime}$ : instead we will consider as unknown $\tau_{0}^{\prime}=\tau^{\prime}-\widetilde{T}$ on $\Gamma_{0}^{u}$. Now thanks to lemma 3.1, $\tau_{0}^{\prime}$ is sought in $H_{00}^{\frac{1}{2}}\left(\Gamma_{0}^{u}\right)$.

The data completion problem $\left(\mathcal{P}_{0}\right)$ is converted to an optimal control problem following the approach used in [5], [1] and [10]. The difference is that now it includes three states instead of two. Each of them represents a boundary condition either on the inaccessible boundary $\Gamma_{a}$ or on the accessible but unobserved boundary $\Gamma_{0}^{u}$. For every triplet $\left(\eta, \tau, \tau_{0}^{\prime}\right) \in\left(H_{00}^{\frac{1}{2}}\left(\Gamma_{a}\right)\right)^{\prime} \times H_{00}^{\frac{1}{2}}\left(\Gamma_{a}\right) \times H_{00}^{\frac{1}{2}}\left(\Gamma_{0}^{u}\right)$, we define the triplet of states $\left(u_{1}, u_{2}, u_{3}\right)$ as follows:

$$
\left(\mathcal{P}_{1}\right)\left\{\begin{array}{ccc}
-\Delta u_{1}=0 & \text { in } \quad \Omega, \\
u_{1}=0 & \text { on } \quad \Sigma, \\
u_{1}=T & \text { on } \quad \Gamma_{0}^{m}, \\
u_{1}=\widetilde{T} & \text { on } \quad \Gamma_{0}^{u}, \\
\frac{\partial u_{1}}{\partial n}=\eta & \text { on } \quad \Gamma_{a},
\end{array}, \quad\left(\mathcal{P}_{2}\right) \quad\left\{\begin{array}{ccc}
-\Delta u_{2}=0 & \text { in } & \Omega, \\
u_{2}=0 & \text { on } & \Sigma, \\
\frac{\partial u_{2}}{\partial n}=0 & \text { on } & \Gamma_{0}, \\
u_{2}=\tau & \text { on } & \Gamma_{a},
\end{array}\right.\right.
$$

and

$$
\left(\mathcal{P}_{3}\right)\left\{\begin{array}{ccc}
-\Delta u_{3}=0 & \text { in } & \Omega \\
u_{3}=0 & \text { on } & \Sigma, \\
u_{3}=0 & \text { on } & \Gamma_{0}^{m} \\
u_{3}=\tau_{0}^{\prime}=\tau^{\prime}-\widetilde{T} & \text { on } & \Gamma_{0}^{u} \\
\frac{\partial u_{3}}{\partial n}=0 & \text { on } & \Gamma_{a}
\end{array}\right.
$$

where $\eta, \tau$ and $\tau_{0}^{\prime}$ are the control variables. The three states are well defined in $H^{1}(\Omega)$.

We define the following cost functional depending on the control variables:

$$
J\left(\eta, \tau, \tau_{0}^{\prime}\right)=\int_{\Omega}\left|\left(\nabla u_{1}+\nabla u_{3}\right)-\nabla u_{2}\right|^{2} d x d y
$$


We minimize this functional with respect to $\eta, \tau$ and $\tau_{0}^{\prime}$ in $\left(H_{00}^{\frac{1}{2}}\left(\Gamma_{a}\right)\right)^{\prime} \times H_{00}^{\frac{1}{2}}\left(\Gamma_{a}\right) \times H_{00}^{\frac{1}{2}}\left(\Gamma_{0}^{u}\right)$. Owing to the assumption of perfect observation on $\Gamma_{0}^{m}$ we made, we have that the minimum of $J$ is 0 :

$$
\inf _{\left(\eta, \tau, \tau_{0}^{\prime}\right) \in\left(H_{00}^{\frac{1}{2}}\left(\Gamma_{a}\right)\right)^{\prime} \times H_{00}^{\frac{1}{2}}\left(\Gamma_{a}\right) \times H_{00}^{\frac{1}{2}}\left(\Gamma_{0}^{u}\right)} J\left(\eta, \tau, \tau_{0}^{\prime}\right)=J\left(\varphi, t, \hat{\tau}^{\prime}-\widetilde{T}\right)=0 .
$$

Clearly, at the minimum, $u_{1}(\varphi)+u_{3}\left(\hat{\tau^{\prime}}-\widetilde{T}\right)=u_{2}(t)$. We denote $\Delta_{\Gamma}$ the $(n-1) D$-laplacian on any section $\Gamma_{x}$. The spaces $\left(H_{00}^{\frac{1}{2}}\left(\Gamma_{a}\right)\right)^{\prime}$ and $H_{00}^{\frac{1}{2}}\left(\Gamma_{a}\right)$ are equipped, respectively, with the following inner product (see [21]):

$$
\langle u, v\rangle\left(H_{00}^{\frac{1}{2}}\left(\Gamma_{a}\right)\right)^{\prime}=\int_{\Gamma_{a}}\left(-\Delta_{\Gamma}\right)^{-\frac{1}{4}} u\left(-\Delta_{\Gamma}\right)^{-\frac{1}{4}} v d y=\int_{\Gamma_{a}} u\left(-\Delta_{\Gamma}\right)^{-\frac{1}{2}} v d y,
$$

for all $u, v \in\left(H_{00}^{\frac{1}{2}}\left(\Gamma_{a}\right)\right)^{\prime}$, and

$$
\langle u, v\rangle_{H_{00}^{\frac{1}{2}}\left(\Gamma_{a}\right)}=\int_{\Gamma_{a}}\left(-\Delta_{\Gamma}\right)^{\frac{1}{4}} u\left(-\Delta_{\Gamma}\right)^{\frac{1}{4}} v d y=\int_{\Gamma_{a}} u\left(-\Delta_{\Gamma}\right)^{\frac{1}{2}} v d y
$$

for all $u, v \in H_{00}^{\frac{1}{2}}\left(\Gamma_{a}\right)$. Similarly

$$
\langle u, v\rangle_{H_{00}^{\frac{1}{2}}\left(\Gamma_{0}^{u}\right)}=\int_{\Gamma_{0}^{u}}\left(-\Delta_{\Gamma}\right)^{\frac{1}{4}} u\left(-\Delta_{\Gamma}\right)^{\frac{1}{4}} v d y=\int_{\Gamma_{0}^{u}} u\left(-\Delta_{\Gamma}\right)^{\frac{1}{2}} v d y
$$

for all $u, v \in H_{00}^{\frac{1}{2}}\left(\Gamma_{0}^{u}\right)$. Due to the ill-posedness of the inverse problem, and in particular because of the noncontinuity of solutions with respect to the Cauchy data [14] the resolution with noisy observations requires the use of a regularization. We use a Tikhonov regularization and denote by $J_{\epsilon}$ the new cost function:

$$
J_{\epsilon}\left(\eta, \tau, \tau_{0}^{\prime}\right)=J\left(\eta, \tau, \tau_{0}^{\prime}\right)+\epsilon\left(\|\eta\|_{\left(H_{00}^{\frac{1}{2}}\left(\Gamma_{a}\right)\right)^{\prime}}^{2}+\|\tau\|_{H_{00}^{\frac{1}{2}}\left(\Gamma_{a}\right)}^{2}+\left\|\tau_{0}^{\prime}\right\|_{H_{00}^{\frac{1}{2}}\left(\Gamma_{0}^{u}\right)}^{2}\right) .
$$

where $\epsilon$ is a non negative regularization parameter. One could use a different parameter for each of the regularization terms. Here, we use the same parameter $\epsilon$ just for the sake of simplicity.

\section{BRIEF SKETCH OF THE FACTORIZATION METHOD FOR BOUNDARY VALUE PROBLEMS}

In this section, we show how to apply the factorization method to the states $u_{1}, u_{2}$ and $u_{3}$. In the next section we will show we can express the cost function explicitly in terms of the controls $\eta, \tau$ and $\tau_{0}^{\prime}$ using the operators derived in this section for the factorization. The technique of invariant embedding appeared in the field of the theory of optimal control with Bellman, [9]. The method developed in [16-18] allows to use the factorization approach to solve elliptic boundary value problems. This method is also used in [19] and [1] for solving a data completion problem in a cylindrical domain. Recently this method has been used to solve the inverse problem in electrocardiography for realistic heart and torso geometry [10]. We embed the boundary value problem for the state equation in a family of similar problems defined on $\Omega_{s}$ sub-domains of $\Omega$. For that we define $\Gamma_{s}=\{s\} \times \mathcal{O}$ a mobile boundary which will move from $s=0$ to $s=a$. At each position $s$, one can thus define a sub-domain $\left.\Omega_{s}=\right] 0, s\left[\times \mathcal{O}\right.$ with lateral boundary $\Sigma_{s}$ delimited by surfaces $\Gamma_{0}$ and $\Gamma_{s}$. The schematic representation of $\Omega_{s}$ is given in Figure 2. The general formulation for non cylindrical geometries could be found in [18]. 


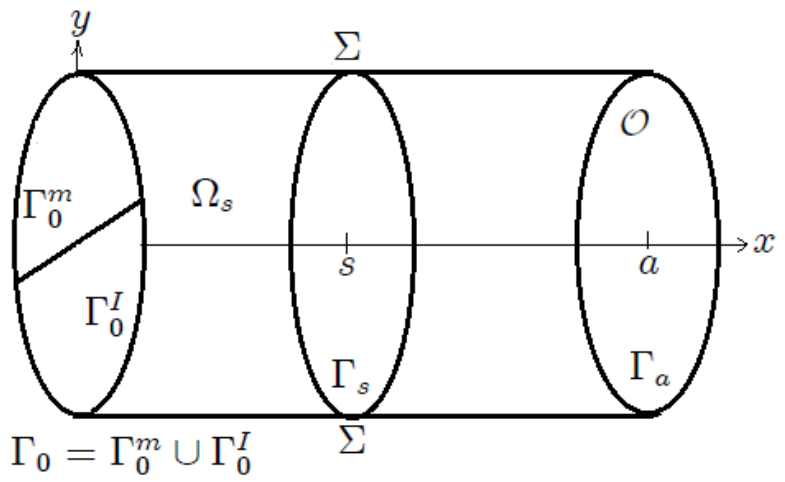

FIGURE 2. Schematic representation of the moving domain $\Omega_{s}$.

For given boundary data $\left(\alpha_{1}, \beta, \alpha_{2}\right) \in\left(H_{00}^{\frac{1}{2}}\left(\Gamma_{s}\right)\right)^{\prime} \times H_{00}^{\frac{1}{2}}\left(\Gamma_{s}\right) \times H_{00}^{\frac{1}{2}}\left(\Gamma_{0}^{u}\right)$, we define $u_{1}^{s}, u_{2}^{s}$ and $u_{3}^{s}$ solution of the following three problems in $\Omega_{s}$ :

$$
\left(\mathcal{P}_{1}^{s}\right)\left\{\begin{array} { c c c } 
{ - \Delta u _ { 1 } ^ { s } = 0 } & { \text { in } \quad \Omega _ { s } , } \\
{ u _ { 1 } ^ { s } = 0 } & { \text { on } \quad \Sigma _ { s } , } \\
{ u _ { 1 } ^ { s } = T } & { \text { on } \quad \Gamma _ { 0 } ^ { m } , } \\
{ u _ { 1 } ^ { s } = \widetilde { T } } & { \text { on } \quad \Gamma _ { 0 } ^ { u } , } \\
{ \frac { \partial u _ { 1 } ^ { s } } { \partial n } = \alpha _ { 1 } } & { \text { on } \quad \Gamma _ { s } , }
\end{array} \quad ( \mathcal { P } _ { 2 } ^ { s } ) \left\{\begin{array}{cl}
-\Delta u_{2}^{s}=0 & \text { in } \Omega_{s}, \\
u_{2}^{s}=0 & \text { on } \Sigma_{s}, \\
\frac{\partial u_{2}^{s}}{\partial n}=0 & \text { on } \Gamma_{0}, \\
u_{2}^{s}=\beta & \text { on } \Gamma_{s},
\end{array}\right.\right.
$$

and

where $n$ is the outward normal to $\Omega_{s}$.

$$
\left(\mathcal{P}_{3}^{s}\right)\left\{\begin{array}{ccc}
-\Delta u_{3}^{s}=0 & \text { in } & \Omega_{s}, \\
u_{3}^{s}=0 & \text { on } & \Sigma_{s}, \\
u_{3}^{s}=0 & \text { on } & \Gamma_{0}^{m}, \\
u_{3}^{s}=\alpha_{2} & \text { on } & \Gamma_{0}^{u}, \\
\frac{\partial u_{3}^{s}}{\partial n}=0 & \text { on } & \Gamma_{s},
\end{array}\right.
$$

\subsection{Neumann-Dirichlet mapping}

By splitting problem $\left(\mathcal{P}_{1}^{s}\right)$ into two well posed problems, we can write $u_{1}^{s}$ as a sum of two functions $\gamma_{s}$ and $\delta_{s}$ depending linearly on $\alpha_{1}$ and $T$ respectively. The functions $\gamma_{s}$ and $\delta_{s}$ are solutions of the following two problems:

$$
\left\{\begin{array} { c c c } 
{ - \Delta \gamma _ { s } = 0 } & { \text { in } \quad \Omega _ { s } , } \\
{ \gamma _ { s } = 0 } & { \text { on } \quad \Sigma _ { s } , } \\
{ \gamma _ { s } = 0 } & { \text { on } \Gamma _ { 0 } ^ { m } , } \\
{ \gamma _ { s } = 0 } & { \text { on } \quad \Gamma _ { 0 } ^ { u } , } \\
{ \frac { \partial \gamma _ { s } } { \partial n } = \alpha _ { 1 } } & { \text { on } \quad \Gamma _ { s } , }
\end{array} \quad \text { and } \quad \left\{\begin{array}{ccc}
-\Delta \delta_{s}=0 & \text { in } \quad \Omega_{s}, \\
\delta_{s}=0 & \text { on } \quad \Sigma_{s}, \\
\delta_{s}=T & \text { on } & \Gamma_{0}^{m} \\
\delta_{s}=\widetilde{T} & \text { on } & \Gamma_{0}^{u} \\
\frac{\partial \delta_{s}}{\partial n}=0 & \text { on } & \Gamma_{s}
\end{array}\right.\right.
$$


For every $s \in] 0, a]$ we define the Neumann-Dirichlet mapping $Q(s)$ by:

$$
Q(s) \alpha_{1}=\left.\gamma_{s}\right|_{\Gamma_{s}} .
$$

We also define the residual part $\omega(s)$ associated to the operator $Q$ by $\omega(s)=\delta_{s} \mid \Gamma_{s} \in H_{00}^{\frac{1}{2}}\left(\Gamma_{s}\right)$. We have:

$$
\left.u_{1}^{s}\right|_{\Gamma_{s}}=Q(s) \alpha_{1}+\omega(s) .
$$

For every $s$ in $] 0, a], Q(s):\left(H_{00}^{\frac{1}{2}}\left(\Gamma_{s}\right)\right)^{\prime} \mapsto H_{00}^{\frac{1}{2}}\left(\Gamma_{s}\right)$ is a coercive, self-adjoint, linear continuous operator. For $x<s$ that the restriction of $u_{1}^{s}\left(\alpha_{1}\right)$ on $\Omega_{x}$, is a solution of the problem $\left(\mathcal{P}_{1}^{x}\right)$. By the same calculation as previously we have the relation:

$$
u_{1}^{s}\left(x, \alpha_{1}\right)=Q(x) \frac{\partial u_{1}^{s}}{\partial x}\left(x, \alpha_{1}\right)+\omega(x) .
$$

For the sake of clarity, from now on, we denote by $u_{1}$ (respectively, $Q$ and $\omega$ ), the restriction on $\Gamma_{x} u_{1}(x)$ (respectively, $Q(x)$ and $\omega(x)$ ):

$$
u_{1}=Q \frac{\partial u_{1}}{\partial x}+\omega
$$

In a formal way, we take the derivative of (4.4) with respect to $x$ :

$$
\frac{d u_{1}}{d x}=\frac{d Q}{d x} \frac{d u_{1}}{d x}+Q \frac{d^{2} u_{1}}{d x^{2}}+\frac{d \omega}{d x} .
$$

By replacing the term $\frac{d^{2} u_{1}}{d x^{2}}$ by $-\Delta_{\Gamma} u_{1}$ and substituting $u_{1}$, using (4.4) and the fact that $\alpha_{1}$ is arbitrary we get:

$$
\left\{\begin{array}{l}
\frac{d Q}{d x}-Q \Delta_{\Gamma} Q=I \\
\frac{d \omega}{d x}-Q \Delta_{\Gamma} \omega=0
\end{array}\right.
$$

One can extend by continuity this argument to $x=0$. Writing relation (4.3) at $x=0$, and by using the fact that $u_{1}(0)$ is arbitrary, we get the initial conditions $\left.Q\right|_{\Gamma_{0}}=0,\left.\omega\right|_{\Gamma_{0}^{m}}=T$ and $\left.\omega\right|_{\Gamma_{0}^{u}}=\tilde{T}$. Adding the condition on $\Gamma_{a}$ for $u_{1}$, we summarize the result in an equivalent formulation of $\left(\mathcal{P}_{1}\right)$ :

$$
\begin{cases}\frac{d Q}{d x}-Q \Delta_{\Gamma} Q=I & Q_{\mid \Gamma_{0}}=0, \\ \frac{d \omega}{d x}-Q \Delta_{\Gamma} \omega=0 & \left.\omega\right|_{\Gamma_{0}^{m}}=T,\left.\quad \omega\right|_{\Gamma_{0}^{u}}=\tilde{T}, \\ u_{1}=Q \frac{\partial u_{1}}{\partial x}+\omega & \left.\frac{\partial u_{1}}{\partial x}\right|_{\Gamma_{a}}=\eta .\end{cases}
$$

\subsection{Dirichlet-Neumann mapping}

We use the same methodology as in the previous paragraph. We define the Dirichlet-Neumann mapping $P(s)$ as the linear operator that maps $\beta$ to $\left.\frac{\partial u_{2}^{s}}{\partial x}\right|_{\Gamma_{s}}$. Again, for all $x<s$ the restriction $u_{2}^{s}(x)$ satisfies:

$$
\frac{\partial u_{2}^{s}}{\partial x}(x)=P(x) u_{2}^{s}(x),
$$

or simply:

$$
\frac{\partial u_{2}^{s}}{\partial x}=P u_{2}^{s}
$$


Taking the derivative with respect to $x$ :

$$
\frac{d^{2} u_{2}^{s}}{d x^{2}}=\frac{d P}{d x} u_{2}^{s}+P \frac{\partial u_{2}^{s}}{\partial x}
$$

and substituting $u_{2}^{s}$, using $\left(\mathcal{P}_{2}^{s}\right)$, taking $x=s$ and using the fact that $\beta$ is arbitrary we deduce:

$$
\frac{d P}{d x}+P^{2}=-\Delta_{\Gamma}
$$

We can extend by continuity this argument to $x=0$. Using the fact that $u_{2}(0)$ is arbitrary and that the flux of $u_{2}$ over $\Gamma_{0}$ is equal to zero, we get the initial conditions for $P,\left.\quad P\right|_{\Gamma_{0}}=0$. Adding the condition on $\Gamma_{a}$ for $u_{2}$, we summarize the result: an equivalent formulation of $\left(\mathcal{P}_{2}\right)$ is:

$$
\left\{\begin{array}{cc}
\frac{d P}{d x}+P^{2}=-\Delta_{\Gamma} & \left.P\right|_{\Gamma_{0}}=0, \\
\frac{\partial u_{2}}{\partial x}=P u_{2} & \left.u_{2}\right|_{\Gamma_{a}}=\tau .
\end{array}\right.
$$

\subsection{New operators related to the incomplete measurement}

We consider the family of problems $\left(\mathcal{P}_{3}^{s}\right)$. For every $s$ in $\left.] 0, a\right]$ we define a new Dirichlet-Neumann mapping $S$ as the linear operator that maps $\alpha_{2}$ to $\left.\frac{\partial u_{3}^{s}}{\partial x}\right|_{\Gamma_{0}^{u}}$. We note that the operator $S$ is well defined, because for any $\alpha_{2}$ we can solve problem $\left(\mathcal{P}_{3}^{s}\right)$. Then by linearity of the problem $\left(\mathcal{P}_{3}^{s}\right)$, so by the well-posedness of the problem on $u_{3}^{s}$ and the continuity of the trace mapping we define, for every $s$ in $\left.] 0, a\right]$, the operator $S(s)$ as follows:

$$
\begin{aligned}
S(s): \quad H_{00}^{\frac{1}{2}}\left(\Gamma_{0}^{u}\right) \mapsto\left(H_{00}^{\frac{1}{2}}\left(\Gamma_{0}^{u}\right)\right)^{\prime} \\
\left.\alpha_{2} \mapsto \frac{\partial u_{3}^{s}}{\partial s}\left(\alpha_{2}\right)\right|_{\Gamma_{0}^{u}}=S(s) \alpha_{2} .
\end{aligned}
$$

$S(s)$ is a coercive, self-adjoint, linear continuous operator. For further calculations, we simplify the notation by writing:

$$
\frac{\partial u_{3}^{s}}{\partial s}=S u_{3}^{s} .
$$

By defining the extension operator $R$ and projection operators $\Pi_{u}$ and $\Pi_{m}$ :

$$
\begin{aligned}
& R: \quad H_{00}^{\frac{1}{2}}\left(\Gamma_{0}^{u}\right) \mapsto H_{00}^{\frac{1}{2}}\left(\Gamma_{0}\right) \\
& \alpha_{2} \mapsto \alpha_{2}^{0}=\left\{\begin{array}{c}
\alpha_{2} \text { in } \Gamma_{0}^{u}, \\
0 \text { in } \Gamma_{0}^{m},
\end{array}\right. \\
& \Pi_{u}:\left(H_{00}^{\frac{1}{2}}\left(\Gamma_{0}\right)\right)^{\prime} \mapsto\left(H^{\frac{1}{2}}\left(\Gamma_{0}^{u}\right) \cap H_{00}^{\frac{1}{2}}\left(\Gamma_{0}\right)\right)^{\prime} \\
& \left.g \mapsto g\right|_{\Gamma_{0}^{u}}, \\
& \Pi_{m}: \quad\left(H_{00}^{\frac{1}{2}}\left(\Gamma_{0}\right)\right)^{\prime} \mapsto\left(H^{\frac{1}{2}}\left(\Gamma_{0}^{m}\right) \cap H_{00}^{\frac{1}{2}}\left(\Gamma_{0}\right)\right)^{\prime} \\
& \left.g \mapsto g\right|_{\Gamma_{0}^{m}},
\end{aligned}
$$

and using the symmetry transformation $x \rightarrow a-x$ of the cylindrical domain $\Omega$, we obtain the following relation between the Dirichlet-Neumann operators $S$ and $P$ :

$$
S(s)=\Pi_{u} \circ P(s) \circ R,
$$


where $\circ$ is the composition sign.

We also define for every $s$ in $] 0, a]$ the operator $H$ as follows:

$$
\begin{aligned}
& H(s): \quad H_{00}^{\frac{1}{2}}\left(\Gamma_{0}^{u}\right) \mapsto H_{00}^{\frac{1}{2}}\left(\Gamma_{s}\right) \\
&\left.\alpha_{2} \mapsto u_{3}^{s}\left(\alpha_{2}\right)\right|_{\Gamma_{s}}=H(s) \alpha_{2} .
\end{aligned}
$$

The linear operator $H$ could be constructed based on the residual $\omega$ defined in (4.1). In fact, for a given $\alpha_{2} \in H_{00}^{\frac{1}{2}}\left(\Gamma_{0}^{u}\right), H(s) \alpha_{2}$ corresponds to the residual $\omega$ with the initial condition $\omega(0)=R \alpha_{2}$.

The new operator $P^{\prime}$ is defined as follows:

$$
\begin{aligned}
P^{\prime}(s): \quad H_{00}^{\frac{1}{2}}\left(\Gamma_{0}\right) \mapsto\left(H_{00}^{\frac{1}{2}}\left(\Gamma_{0}\right)\right)^{\prime} \\
\left.T_{0} \mapsto \frac{\partial u_{1}^{\prime}}{\partial s}\right|_{\Gamma_{0}}=P^{\prime}(s) T_{0},
\end{aligned}
$$

where $T_{0}=\left\{\begin{array}{c}T \text { on } \Gamma_{0}^{m} \\ \tilde{T} \text { on } \Gamma_{0}^{u}\end{array}\right.$, and $u_{1}^{\prime}$ is the solution of:

$$
\left\{\begin{array}{ccc}
-\Delta u_{1}^{\prime}=0 & \text { in } & \Omega_{s} \\
u_{1}^{\prime}=0 & \text { on } & \Sigma_{s} \\
\frac{\partial u_{1}^{\prime}}{\partial n}=0 & \text { on } & \Gamma_{s} \\
u_{1}^{\prime}=T_{0} & \text { on } & \Gamma_{0}
\end{array}\right.
$$

This operator satisfies:

$$
\frac{d P^{\prime}}{d s}(s)+\left(P^{\prime}\right)^{2}(s)=-\Delta_{\Gamma} \quad P^{\prime}(0)=0 .
$$

By the uniqueness of the solution of the Riccati equation and due the symmetry of the cylindrical domain, $P^{\prime}$ is identical to $P$ but we keep the notation $P^{\prime}$ to recall that we use it on $\Gamma_{0}$.

\section{Solving the optimal Control Problem}

The method of invariant embedding used in the factorization of the state equations is of the same nature as the one used to factorize optimal control problems. Indeed, the energy functional $J$ can be expressed directly in terms of the control variables $\eta, \tau$ and $\tau^{\prime}$ using the operators $P, Q, S$ and $H$. So there is no need to introduce an adjoint state to derive the optimality condition. Saying it in another way, the classical decoupling of the optimality system between state and adjoint state using a Riccati equation is performed here at the same time as the factorization of the state equation.

From now we will denote $P=P(a), Q=Q(a), S=S(a)$ and $H=H(a)$. Let $A$ be the matrix operator defined as follows

$$
A=\left(\begin{array}{ccc}
Q & -Q P & 0 \\
-P Q & P & -P H \\
0 & -H^{*} P & S
\end{array}\right),
$$


$H^{*}$ is the adjoint operator of $H$. Let us denote $\left[\eta, \tau, \tau_{0}^{\prime}\right]$ the row vector with components $\eta, \tau$ and $\tau_{0}^{\prime}$. Let us remark that in relation to the ill-posedness of the Cauchy problem, the operator $A$ is not coercive, but it is self-adjoint.

Proposition 5.1. The cost functional J can be written equivalently,

$$
J\left(\eta, \tau, \tau_{0}^{\prime}\right)=K+\left(\eta, \tau, \tau_{0}^{\prime}\right) A\left(\begin{array}{c}
\eta \\
\tau \\
\tau_{0}^{\prime}
\end{array}\right)-2\left\langle\omega\left(T_{0}\right), P \tau\right\rangle_{\Gamma_{a}}+2\left\langle\tau_{0}^{\prime}, \Pi_{u} \circ P^{\prime} T_{0}\right\rangle_{\Gamma_{0}^{u}}
$$

where $K$ is a constant that does not depend on $\eta, \tau$ and $\tau_{0}^{\prime}$. If a minimum of $J$ is reached at the triplet $\left(\varphi, t, t^{\prime}\right)$, it satisfies:

$$
A\left[\varphi, t, t^{\prime}\right]^{\prime}=\left[0, P \omega\left(T_{0}\right),-\Pi_{u} \circ P^{\prime} T_{0}\right]^{\prime} .
$$

Proof 5.1. Let $\lambda_{1}, \lambda_{2}$ be the solutions of:

$$
\left\{\begin{array}{ccc}
-\Delta \lambda_{1}=0 & \text { in } & \Omega, \\
\lambda_{1}=T_{0} & \text { on } & \Gamma_{0}, \\
\frac{\partial \lambda_{1}}{\partial n}=0 & \text { on } & \Gamma_{a} .
\end{array}, \quad\left\{\begin{array}{cccc}
-\Delta \lambda_{2}=0 & \text { in } & \Omega \\
\lambda_{2}=\tau_{0}^{\prime} & \text { on } & \Gamma_{0} \\
\frac{\partial \lambda_{2}}{\partial n}=0 & \text { on } & \Gamma_{a}
\end{array}\right.\right.
$$

and $\lambda=\lambda_{1}+\lambda_{2}$ satisfies

$$
\left\{\begin{array}{ccc}
-\Delta \lambda=0 & \text { in } & \Omega \\
\lambda=T^{\prime} & \text { on } & \Gamma_{0} \\
\frac{\partial \lambda}{\partial n}=0 & \text { on } & \Gamma_{a}
\end{array}\right.
$$

where $T^{\prime}=\left\{\begin{array}{l}T \text { on } \Gamma_{0}^{m} \\ \tau^{\prime} \text { on } \Gamma_{0}^{u}\end{array}\right.$

To alleviate notations, we will abusively denote duality pairings with integrals. We set $v_{1}=u_{1}+u_{3}$, applying Green's formula to cost functional (3.1) then:

$$
\begin{aligned}
J\left(\eta, \tau, \tau_{0}^{\prime}\right) & =\int_{\Omega}\left|\nabla v_{1}-\nabla u_{2}\right|^{2} d x d y \\
& =\int_{\Gamma_{0}} \frac{\partial v_{1}}{\partial n}\left(T^{\prime}-u_{2}\right) d y+\int_{\Gamma a}\left(\eta-\frac{\partial u_{2}}{\partial n}\right)\left(v_{1}-\tau\right) d y
\end{aligned}
$$

Using the full Green's formula for the term in $\frac{\partial v_{1}}{\partial n} u_{2}$

$$
J\left(\eta, \tau, \tau_{0}^{\prime}\right)=\int_{\Gamma_{0}} \frac{\partial v_{1}}{\partial n} \lambda d y+\int_{\Gamma_{a}}\left(\eta \tau-v_{1} \frac{\partial u_{2}}{\partial n}\right) d y+\int_{\Gamma a}\left(\eta-\frac{\partial u_{2}}{\partial n}\right)\left(v_{1}-\tau\right) d y
$$


and for the terms in $\frac{\partial v_{1}}{\partial n}$

$$
J\left(\eta, \tau, \tau_{0}^{\prime}\right)=\int_{\Gamma_{0}} T^{\prime} \nabla \lambda \cdot n d y+\int_{\Gamma_{a}}\left(\eta\left(v_{1}-\lambda\right)+\frac{\partial u_{2}}{\partial n} \tau\right) d y-2 \int_{\Gamma a} v_{1} \frac{\partial u_{2}}{\partial n} d y .
$$

From (4.4), (4.6) and from the definition of $\lambda(\lambda(a)=\omega(a))$ we obtain

$$
J\left(\eta, \tau, \tau_{0}^{\prime}\right)=\int_{\Gamma_{0}} T^{\prime} \frac{\partial \lambda}{\partial n} d y+\int_{\Gamma_{a}}(\eta Q \eta+\tau P \tau) d y-2 \int_{\Gamma a}\left(Q \eta+\omega\left(T^{\prime}\right)\right) P \tau d y
$$

From the definition of $T^{\prime}$ and $\lambda$ we have:

$$
\int_{\Gamma_{0}} T^{\prime} \frac{\partial \lambda}{\partial n} d y=\int_{\Gamma_{0}^{m}} T \frac{\partial \lambda_{1}}{\partial n} d \Gamma_{0}^{m}+\int_{\Gamma_{0}^{u}} \tau^{\prime} \frac{\partial \lambda_{1}}{\partial n} d \Gamma_{0}^{u}+\int_{\Gamma_{0}^{m}} T \frac{\partial \lambda_{2}}{\partial n} d \Gamma_{0}^{m}+\int_{\Gamma_{0}^{u}} \tau^{\prime} \frac{\partial \lambda_{2}}{\partial n} d \Gamma_{0}^{u}
$$

where the term $K:=\int_{\Gamma_{0}^{m}} T \frac{\partial \lambda_{1}}{\partial n} d \Gamma_{0}^{m}$ does not depend on controls $\eta, \tau$ and $\tau_{0}^{\prime}$. According to the definition of operators $S, P^{\prime}, \Pi_{m}$ and $\Pi_{u}$, we have

$$
\left.\frac{\partial \lambda_{2}}{\partial n}\right|_{\Gamma_{0}^{u}}=S \tau_{0}^{\prime},\left.\quad \frac{\partial \lambda_{1}}{\partial n}\right|_{\Gamma_{0}^{u}}=\Pi_{u} \circ P^{\prime} T_{0},\left.\quad \frac{\partial \lambda_{2}}{\partial n}\right|_{\Gamma_{0}^{m}}=\Pi_{m} \circ P^{\prime} \tau_{0}^{\prime},
$$

We denote $\omega\left(T^{\prime}\right)$ by $\omega\left(T ; \tau^{\prime}\right)$ in order to show the dependency of $\omega$ on the data on $\Gamma_{0}^{m}$ and $\Gamma_{0}^{u}$. We have:

$$
\omega\left(T^{\prime}\right)=\omega\left(T ; \tau^{\prime}\right)+\omega\left(0 ; \tau_{0}^{\prime}\right)=\omega\left(T_{0}\right)+H \tau_{0}^{\prime}
$$

Substituting (5.15), (5.16) and (5.17) in (5.14) we obtain

$$
\begin{aligned}
J\left(\eta, \tau, \tau_{0}^{\prime}\right)= & K+\int_{\Gamma_{0}^{u}} \tau^{\prime} \Pi_{u} \circ P^{\prime} T_{0} d \Gamma_{0}^{u}+\int_{\Gamma_{0}^{m}} T \Pi_{m} \circ P^{\prime} \tau_{0}^{\prime} d \Gamma_{0}^{m}+\int_{\Gamma_{0}^{u}} \tau^{\prime} S \tau_{0}^{\prime} d \Gamma_{0}^{u} \\
& +\int_{\Gamma_{a}}(\eta Q \eta+\tau P \tau) d \Gamma_{a}-2 \int_{\Gamma_{a}}\left(Q \eta+\omega\left(T_{0}\right)\right) P \tau d \Gamma_{a} \\
& -2 \int_{\Gamma_{a}} H \tau_{0}^{\prime} P \tau d \Gamma_{a} .
\end{aligned}
$$


The third right-hand side term could be developed as follows

$$
\begin{aligned}
\int_{\Gamma_{0}^{m}} T \Pi_{m} \circ P^{\prime} \tau_{0}^{\prime} d \Gamma_{0}^{m} & =\int_{\Gamma_{0}^{m}} u_{1}^{\prime} \frac{\partial \lambda_{2}}{\partial n} d \Gamma_{0}^{m} \\
& =-\int_{\Gamma_{0}^{u}} u_{1}^{\prime} \frac{\partial \lambda_{2}}{\partial n} d \Gamma_{0}^{u}+\int_{\Omega} \nabla u_{1}^{\prime} \nabla \lambda_{2} d \Omega \\
& =-\int_{\Gamma_{0}^{u}} \tilde{T} S \tau_{0}^{\prime} d \Gamma_{0}^{u}+\int_{\Gamma_{0}^{u}} \frac{\partial u_{1}^{\prime}}{\partial n} \lambda_{2} d \Gamma_{0}^{u} \\
& =-\int_{\Gamma_{0}^{u}} \tilde{T} S \tau_{0}^{\prime} d \Gamma_{0}^{u}+\int_{\Gamma_{0}^{u}} \tau_{0}^{\prime} \Pi_{u} \circ P^{\prime} T_{0} d \Gamma_{0}^{u}
\end{aligned}
$$

By substituting (5.19) in (5.18), we obtain

$$
\begin{aligned}
J\left(\eta, \tau, \tau_{0}^{\prime}\right)= & K^{\prime}+\int_{\Gamma_{0}^{u}} \tau_{0}^{\prime} \Pi_{u} \circ P^{\prime} T_{0} d \Gamma_{0}^{u}+\int_{\Gamma_{0}^{u}} \tau_{0}^{\prime} \Pi_{u} \circ P^{\prime} T_{0} d \Gamma_{0}^{u}+\int_{\Gamma_{0}^{u}} \tau_{0}^{\prime} S \tau_{0}^{\prime} d \Gamma_{0}^{u}+ \\
& \int_{\Gamma_{a}}(\eta Q \eta+\tau P \tau) d \Gamma_{a}-2 \int_{\Gamma_{a}}\left(Q \eta+\omega\left(T_{0}\right)\right) P \tau d \Gamma_{a}-2 \int_{\Gamma_{0}^{u}} \tau_{0}^{\prime} H^{*} P \tau d \Gamma_{0}^{u},
\end{aligned}
$$

where we set $K^{\prime}=K-\int_{\Gamma_{0}^{u}} \widetilde{T} \Pi_{u} \circ P^{\prime} T_{0} d \Gamma_{0}^{u}$. This formula can be written as (5.11) taking into account that $P, Q, S$ are self-adjoint.

Deriving the functional $J$ with respect to $\eta, \tau$ and $\tau_{0}^{\prime}$, we obtain

$$
\begin{aligned}
\left\langle\frac{\partial J}{\partial \eta}, \psi\right\rangle & =2 \int_{\Gamma_{a}} Q \eta \psi d \Gamma_{a}-2 \int_{\Gamma_{a}} Q P \tau, \psi d \Gamma_{a} \\
\left\langle\frac{\partial J}{\partial \tau}, \varphi\right\rangle & =2 \int_{\Gamma_{a}} P \tau \varphi d \Gamma_{a}-2 \int_{\Gamma_{a}} P Q \eta \varphi d \Gamma_{a}-2 \int_{\Gamma_{a}} P H \tau_{0}^{\prime} \varphi d \Gamma_{a}-2 \iint_{\Gamma_{a}} P \omega\left(T_{0}\right) \varphi d \Gamma_{a}, \\
\left\langle\frac{\partial J}{\partial \tau_{0}^{\prime}}, \mu\right\rangle & =2 \int_{\Gamma_{0}^{u}} \Pi_{u} \circ P^{\prime} T_{0} \mu d \Gamma_{0}^{u}+2 \int_{\Gamma_{0}^{u}} S \tau_{0}^{\prime} \mu d \Gamma_{0}^{u}-2 \int_{\Gamma_{0}^{u}} H^{*} P \tau \mu d \Gamma_{0}^{u} .
\end{aligned}
$$

Thus, when the observation on $\Gamma_{0}^{m}$ is exact, the optimum of $J$ is reached at $\left(\varphi, t, t_{0}^{\prime}\right)$ satisfying the following linear system

$$
\begin{aligned}
Q \varphi-Q P t & =0 \\
-P Q \varphi+P t-P H t_{0}^{\prime} & =P \omega\left(T_{0}\right) \\
-H^{*} P t+S t_{0}^{\prime} & =-\Pi_{u} \circ P^{\prime} T_{0} .
\end{aligned}
$$

\section{COMPARISON BETWEEN CASES OF COMPLETE AND INCOMPLETE OBSERVATIONS}

In this section, we will analyse the effect of regularization and noise on the solution of the data completion problem both when considering complete and incomplete observation on the accessible boundary. 


\subsection{Incomplete observation}

In this paragraph, we present an analysis of the Tikhonov regularization in the cylindrical case where the observation of the electrical potential is performed on a part of the accessible boundary $\Gamma_{0}^{m} \subset \Gamma_{0}$ as previously presented. The goal is to estimate how the error between the exact and the regularized problem solution behaves with respect to the size of $\Gamma_{0}^{m}$ and the introduced noise $(u=T+\delta)$. We follow the approach developed by Morozov and Stessin [22]. We recall that the cost function with the Tikhonov regularization term defined in $(3.2)$ is

$$
\left.J_{\epsilon}\left(\eta, \tau, \tau_{0}^{\prime}\right)=J\left(\eta, \tau, \tau_{0}^{\prime}\right)+\epsilon\left(\|\eta\|_{\left(H_{00}^{\frac{1}{2}}\left(\Gamma_{a}\right)\right.}^{2}\right)^{\prime}+\|\tau\|_{H_{00}^{\frac{1}{2}}\left(\Gamma_{a}\right)}^{2}+\left\|\tau_{0}^{\prime}\right\|_{H_{00}^{\frac{1}{2}}\left(\Gamma_{0}^{u}\right)}^{2}\right)
$$

Thus, from Proposition 5.1, the optimum of $J_{\epsilon}$ is reached at $\left(\varphi_{\epsilon}, t_{\epsilon}, t_{\epsilon}^{\prime}\right)$ satisfying the following linear system

$$
\begin{aligned}
(\epsilon I+Q) \varphi_{\epsilon}-Q P t_{\epsilon} & =0 \\
-P Q \varphi_{\epsilon}+(\epsilon I+P) t_{\epsilon}-P H t_{\epsilon}^{\prime} & =P \omega\left(T_{0}\right), \\
-H^{*} P t_{\epsilon}+(\epsilon I+S) t_{\epsilon}^{\prime} & =-\Pi_{u} \circ P^{\prime} T_{0} .
\end{aligned}
$$

Denoting by $A_{\epsilon}=A+\epsilon I$, we have

$$
A_{\epsilon}\left[\varphi_{\epsilon}, t_{\epsilon}, t_{\epsilon}^{\prime}\right]^{\prime}=\left[0, P \omega\left(T_{0}\right),-\Pi_{u} \circ P^{\prime} T_{0}\right]^{\prime} .
$$

Adding a noise $\delta \in L^{2}\left(\Gamma_{0}^{m}\right)$ to the exact observable data $T$, we denote by $\left(\varphi_{\epsilon}^{\delta}, t_{\epsilon}^{\delta}, t_{\epsilon}^{\prime \delta}\right)$, the solution of the regularized problem but with noisy observation $T+\delta$. Obviously, this triplet satisfies

$$
A_{\epsilon}\left[\varphi_{\epsilon}^{\delta}, t_{\epsilon}^{\delta}, t_{\epsilon}^{\delta}\right]^{\prime}=\left[0, P \omega\left(T_{0}+\delta\right),-\Pi_{u} \circ P^{\prime}\left(T_{0}+\delta\right)\right]^{\prime} .
$$

In what follows our aim is twofold. First, we would like to estimate the error between $t_{\epsilon}^{\delta}$ and the exact solution $t$ at the inaccessible boundary $\Gamma_{a}$. Second we want to quantify the effect of the size of $\Gamma_{0}^{m}$ on the error $\left\|t_{\epsilon}^{\delta}-t\right\|_{L^{2}\left(\Gamma_{a}\right)}$.

Following the Morozov's approach, we first apply a Cholesky factorization to the operator $A$.

$$
A=R^{*} R \quad \text { where } R=\left(\begin{array}{ccc}
B & C & 0 \\
0 & D & E \\
0 & 0 & F
\end{array}\right) .
$$

By identification, we have

$$
\left\{\begin{array}{l}
B=Q^{\frac{1}{2}} \\
C=-Q^{\frac{1}{2}} P \\
D=(P-P Q P)^{\frac{1}{2}} \\
E=-(P-P Q P)^{-\frac{1}{2}} P H \\
F=\left[S-H^{*} P(P-P Q P)^{-1} P H\right]^{\frac{1}{2}} .
\end{array}\right.
$$

Theorem 6.1. There exist a decaying positive sequence $\left(h_{\epsilon}\right)_{\epsilon>0}$

$$
h_{\epsilon} \longrightarrow 0 \text { for } \epsilon \longrightarrow 0 \text {, }
$$


a non negative constant $k_{1}$ that depends only on the domain $\Omega$ and a positive constant $k_{2}$ depending on $\Gamma_{0}^{u}$, such that

$$
\left\|t_{\epsilon}^{\delta}-t\right\|_{H_{00}^{\frac{1}{2}\left(\Gamma_{a}\right)}}+\left\|t_{\epsilon}^{\prime \delta}-t^{\prime}\right\|_{H^{\frac{1}{2}}\left(\Gamma_{0}^{u}\right)} \leq \frac{\sqrt{2\left(k_{1}^{2}+k_{2}^{2}\right)}}{\epsilon}\|\delta\|_{L^{2}\left(\Gamma_{0}^{m}\right)}+h_{\epsilon} .
$$

Moreover, if there exist $\left(y_{1}, y_{2}\right) \in L^{2}\left(\Gamma_{a}\right)$ and $y_{3} \in L^{2}\left(\Gamma_{0}^{u}\right)$ such that $t=C^{*} y_{1}+D^{*} y_{2}, \varphi=B^{*} y_{1}$ and $t^{\prime}=E^{*} y_{2}+F^{*} y_{3}$, then the solution satisfies

$$
\left\|t_{\epsilon}^{\delta}-t\right\|_{H_{00}^{\frac{1}{2}}\left(\Gamma_{a}\right)}+\left\|t_{\epsilon}^{\prime \delta}-t^{\prime}\right\|_{H^{\frac{1}{2}}\left(\Gamma_{0}^{u}\right)} \leq \frac{\sqrt{2\left(k_{1}^{2}+k_{2}^{2}\right)}}{\epsilon}\|\delta\|_{L^{2}\left(\Gamma_{0}^{m}\right)}+\sqrt{\epsilon} \sqrt{\left\|y_{1}\right\|^{2}+\left\|y_{2}\right\|^{2}+\left\|y_{3}\right\|^{2}}
$$

and

$$
\left\|t_{\epsilon}^{\delta}-t\right\|_{H_{00}^{\frac{1}{2}}\left(\Gamma_{a}\right)}^{2} \leq \frac{\left(k_{1}^{2}+k_{2}^{2}\right)}{\epsilon^{2}}\|\delta\|_{L^{2}\left(\Gamma_{0}^{m}\right)}^{2}+\epsilon\left(\left\|y_{1}\right\|^{2}+\left\|y_{2}\right\|^{2}+\left\|y_{3}\right\|^{2}\right)
$$

Remark 6.2. The condition $\left(y_{1}, y_{2}\right) \in L^{2}\left(\Gamma_{a}\right)$ and $y_{3} \in L^{2}\left(\Gamma_{0}^{u}\right)$ such that $t=C^{*} y_{1}+D^{*} y_{2}, \varphi=B^{*} y_{1}$ and $t^{\prime}=E^{*} y_{2}+F^{*} y_{3}$ is equivalent to the fact that the triplet solution $\left(\varphi, t, t^{\prime}\right)$ belongs to the image of the operator $R^{*}$.

Proof 6.1. In order to prove this theorem, we use the following triangle inequality

$$
\begin{aligned}
\left\|t_{\epsilon}^{\delta}-t\right\|_{H_{00}^{\frac{1}{2}}\left(\Gamma_{a}\right)}+\left\|t_{\epsilon}^{\prime \delta}-t^{\prime}\right\|_{H^{\frac{1}{2}}\left(\Gamma_{0}^{u}\right)} \leq & \left\|t_{\epsilon}^{\delta}-t_{\epsilon}\right\|_{H_{00}^{\frac{1}{2}}\left(\Gamma_{a}\right)}+\left\|t_{\epsilon}-t\right\|_{H_{00}^{\frac{1}{2}}\left(\Gamma_{a}\right)} \\
& +\left\|t_{\epsilon}^{\prime \delta}-t_{\epsilon}^{\prime}\right\|_{H^{\frac{1}{2}}\left(\Gamma_{0}^{u}\right)}+\left\|t_{\epsilon}^{\prime}-t^{\prime}\right\|_{H^{\frac{1}{2}}\left(\Gamma_{0}^{u}\right)} .
\end{aligned}
$$

In what follows, we will estimate each of both $\left(\left\|t_{\epsilon}^{\prime}-t^{\prime}\right\|_{H_{00}^{\frac{1}{2}}\left(\Gamma_{a}\right)}+\left\|t_{\epsilon}-t\right\|_{H^{\frac{1}{2}}\left(\Gamma_{0}^{u}\right)}\right)$ and $\left(\left\|t_{\epsilon}^{\delta}-t_{\epsilon}\right\|_{H_{00}^{\frac{1}{2}}\left(\Gamma_{a}\right)}+\left\|t_{\epsilon}^{\prime \delta}-t_{\epsilon}^{\prime}\right\|_{H^{\frac{1}{2}}\left(\Gamma_{0}^{u}\right)}\right.$ terms separately. For the sake of simplicity and readability we will consider the $\|$.$\| for all the spaces, it refers$ to $\|\cdot\|_{H_{00}^{\frac{1}{2}}\left(\Gamma_{a}\right)}$ for $t_{\epsilon}^{\delta}, t_{\epsilon}$ and $t$, it refers $\|\cdot\|_{H^{\frac{1}{2}}\left(\Gamma_{0}^{u}\right)}$ for $t_{\epsilon}^{\prime \delta}, t_{\epsilon}^{\prime}$ and $t^{\prime}$ it refers to $\|\cdot\|_{\left(H_{00}^{\frac{1}{2}}\left(\Gamma_{a}\right)\right)^{\prime}}$ for $\varphi_{\epsilon}^{\delta}, \varphi_{\epsilon}$ and $\varphi$, it refers to $\|\cdot\|_{L^{2}\left(\Gamma_{0}^{m}\right)}$ for the noise $\delta$ since we only measure on $\Gamma_{0}^{m}$, and it refers to $\|\cdot\|_{L^{2}(\theta)}$ for the functions $y_{1}, y_{2}$ and $\|\cdot\|_{L^{2}\left(\Gamma_{0}^{u}\right)}$ for $y_{3}$.

Estimation of $\left\|t_{\epsilon}^{\delta}-t_{\epsilon}\right\|_{H_{00}^{\frac{1}{2}}\left(\Gamma_{a}\right)}+\left\|t_{\epsilon}^{\prime \delta}-t_{\epsilon}^{\prime}\right\|_{H^{\frac{1}{2}}\left(\Gamma_{0}^{u}\right)}$

We have

$$
\left\langle A_{\epsilon}\left(\begin{array}{c}
\varphi_{\epsilon}^{\delta}-\varphi_{\epsilon} \\
t_{\epsilon}^{\delta}-t_{\epsilon} \\
t_{\epsilon}^{\prime \delta}-t_{\epsilon}^{\prime}
\end{array}\right),\left(\begin{array}{c}
\varphi_{\epsilon}^{\delta}-\varphi_{\epsilon} \\
t_{\epsilon}^{\delta}-t_{\epsilon} \\
t_{\epsilon}^{\prime \delta}-t_{\epsilon}^{\prime}
\end{array}\right)\right\rangle=\left\langle\left(\begin{array}{c}
0 \\
P \omega(\delta) \\
-\Pi_{u} \circ P^{\prime} \delta
\end{array}\right),\left(\begin{array}{c}
\varphi_{\epsilon}^{\delta}-\varphi_{\epsilon} \\
t_{\epsilon}^{\delta}-t_{\epsilon} \\
t_{\epsilon}^{\prime \delta}-t_{\epsilon}^{\prime}
\end{array}\right)\right\rangle
$$

which means

$$
\begin{gathered}
\left\langle A\left(\begin{array}{c}
\varphi_{\epsilon}^{\delta}-\varphi_{\epsilon} \\
t_{\epsilon}^{\delta}-t_{\epsilon} \\
t_{\epsilon}^{\prime \delta}-t_{\epsilon}^{\prime}
\end{array}\right),\left(\begin{array}{c}
\varphi_{\epsilon}^{\delta}-\varphi_{\epsilon} \\
t_{\epsilon}^{\delta}-t_{\epsilon} \\
t_{\epsilon}^{\prime \delta}-t_{\epsilon}^{\prime}
\end{array}\right)\right\rangle+\epsilon\left\|\varphi_{\epsilon}^{\delta}-\varphi_{\epsilon}\right\|^{2}+\epsilon\left\|t_{\epsilon}^{\delta}-t_{\epsilon}\right\|^{2}+\epsilon\left\|t_{\epsilon}^{\prime \delta}-t_{\epsilon}^{\prime}\right\|^{2}= \\
\\
\left\langle\frac{1}{\sqrt{\epsilon}} P \omega(\delta), \sqrt{\epsilon}\left(t_{\epsilon}^{\delta}-t_{\epsilon}\right)\right\rangle-\left\langle\frac{1}{\sqrt{\epsilon}} \Pi_{u} \circ P^{\prime} \delta, \sqrt{\epsilon}\left(t_{\epsilon}^{\prime \delta}-t_{\epsilon}^{\prime}\right)\right\rangle .
\end{gathered}
$$


Since $A$ is symmetric positive, we have

$$
\epsilon\left\|t_{\epsilon}^{\delta}-t_{\epsilon}\right\|^{2}+\epsilon\left\|t_{\epsilon}^{\prime \delta}-t_{\epsilon}^{\prime}\right\|^{2} \leq \frac{\left(k_{1}^{2}+k_{2}^{2}\right)}{2 \epsilon}\|\delta\|^{2}+\frac{\epsilon}{2}\left[\left\|t_{\epsilon}^{\delta}-t_{\epsilon}\right\|^{2}+\left\|t_{\epsilon}^{\prime \delta}-t_{\epsilon}^{\prime}\right\|^{2}\right],
$$

where $k_{1}=\|P w\|$ and $k_{2}=\left\|\Pi_{u} \circ P^{\prime}\right\|$. We then obtain

$$
\left\|t_{\epsilon}^{\delta}-t_{\epsilon}\right\|^{2}+\left\|t_{\epsilon}^{\prime \delta}-t_{\epsilon}^{\prime}\right\|^{2} \leq \frac{\left(k_{1}^{2}+k_{2}^{2}\right)}{\epsilon^{2}}\|\delta\|^{2}
$$

We conclude that

$$
\left\|t_{\epsilon}^{\delta}-t_{\epsilon}\right\|+\left\|t_{\epsilon}^{\prime \delta}-t_{\epsilon}^{\prime}\right\| \leq \frac{\sqrt{2\left(k_{1}^{2}+k_{2}^{2}\right)}}{\epsilon}\|\delta\|
$$

Estimation of $\left(\left\|t_{\epsilon}^{\prime}-t^{\prime}\right\|_{H_{00}^{\frac{1}{2}}\left(\Gamma_{a}\right)}+\left\|t_{\epsilon}-t\right\|_{H^{\frac{1}{2}}\left(\Gamma_{0}^{u}\right)}\right)$

We have

$$
A_{\epsilon}\left(\begin{array}{c}
\varphi_{\epsilon} \\
t_{\epsilon} \\
t_{\epsilon}^{\prime}
\end{array}\right)=\left(\begin{array}{c}
0 \\
P \omega\left(T_{0}\right) \\
-\Pi_{u} \circ P^{\prime} T_{0}
\end{array}\right)=A\left(\begin{array}{c}
\varphi_{\epsilon} \\
t_{\epsilon} \\
t_{\epsilon}^{\prime}
\end{array}\right)+\epsilon\left(\begin{array}{c}
\varphi_{\epsilon} \\
t_{\epsilon} \\
t_{\epsilon}^{\prime}
\end{array}\right) \quad ; \quad A\left(\begin{array}{c}
\varphi \\
t \\
t^{\prime}
\end{array}\right)=\left(\begin{array}{c}
0 \\
P \omega\left(T_{0}\right) \\
-\Pi_{u} \circ P^{\prime} T_{0}
\end{array}\right) .
$$

This gives

We then obtain

$$
\left\langle A\left(\begin{array}{c}
\varphi_{\epsilon}-\varphi \\
t_{\epsilon}-t \\
t_{\epsilon}^{\prime}-t^{\prime}
\end{array}\right),\left(\begin{array}{c}
\varphi_{\epsilon}-\varphi \\
t_{\epsilon}-t \\
t_{\epsilon}^{\prime}-t^{\prime}
\end{array}\right)\right\rangle+\epsilon\left\langle\left(\begin{array}{c}
\varphi_{\epsilon} \\
t_{\epsilon} \\
t_{\epsilon}^{\prime}
\end{array}\right),\left(\begin{array}{c}
\varphi_{\epsilon}-\varphi \\
t_{\epsilon}-t \\
t_{\epsilon}^{\prime}-t^{\prime}
\end{array}\right)\right\rangle=0
$$

$$
\left\langle A\left(\begin{array}{c}
\varphi_{\epsilon}-\varphi \\
t_{\epsilon}-t \\
t_{\epsilon}^{\prime}-t^{\prime}
\end{array}\right),\left(\begin{array}{c}
\varphi_{\epsilon}-\varphi \\
t_{\epsilon}-t \\
t_{\epsilon}^{\prime}-t^{\prime}
\end{array}\right)\right\rangle=-\epsilon\left\langle\varphi_{\epsilon}, \varphi_{\epsilon}-\varphi\right\rangle-\epsilon\left\langle t_{\epsilon}, t_{\epsilon}-t\right\rangle-\epsilon\left\langle t_{\epsilon}^{\prime}, t_{\epsilon}^{\prime}-t^{\prime}\right\rangle .
$$

On the other side we have

$$
\begin{aligned}
\left\langle\varphi_{\epsilon}, \varphi_{\epsilon}-\varphi\right\rangle & =\left\|\varphi_{\epsilon}-\varphi\right\|^{2}+\left\langle\varphi, \varphi_{\epsilon}-\varphi\right\rangle \\
\left\langle t_{\epsilon}, t_{\epsilon}-t\right\rangle & =\left\|t_{\epsilon}-t\right\|^{2}+\left\langle t, t_{\epsilon}-t\right\rangle \\
\left\langle t_{\epsilon}^{\prime}, t_{\epsilon}^{\prime}-t^{\prime}\right\rangle & =\left\|t_{\epsilon}^{\prime}-t^{\prime}\right\|^{2}+\left\langle t^{\prime}, t_{\epsilon}^{\prime}-t^{\prime}\right\rangle
\end{aligned}
$$

Denoting by

we have

$$
X_{\epsilon}=\left(\begin{array}{c}
\varphi_{\epsilon}-\varphi \\
t_{\epsilon}-t \\
t_{\epsilon}^{\prime}-t^{\prime}
\end{array}\right)
$$

$$
\left\langle A X_{\epsilon}, X_{\epsilon}\right\rangle+\epsilon\left\|\varphi_{\epsilon}-\varphi\right\|^{2}+\epsilon\left\|t_{\epsilon}-t\right\|^{2}+\epsilon\left\|t_{\epsilon}^{\prime}-t^{\prime}\right\|^{2}=-\epsilon\left\langle\varphi, \varphi_{\epsilon}-\varphi\right\rangle-\epsilon\left\langle t, t_{\epsilon}-t\right\rangle-\epsilon\left\langle t^{\prime}, t_{\epsilon}^{\prime}-t^{\prime}\right\rangle .
$$


Using the positivity of $A$, we have

$$
\frac{\epsilon}{2}\left\|\varphi_{\epsilon}-\varphi\right\|^{2}+\frac{\epsilon}{2}\left\|t_{\epsilon}-t\right\|^{2}+\frac{\epsilon}{2}\left\|t_{\epsilon}^{\prime}-t^{\prime}\right\|^{2} \leq \frac{\epsilon}{2}\left(\|\varphi\|^{2}+\|t\|^{2}+\left\|t^{\prime}\right\|^{2}\right)
$$

Or equivalently

$$
\left\|\varphi_{\epsilon}-\varphi\right\|^{2}+\left\|t_{\epsilon}-t\right\|^{2}+\left\|t_{\epsilon}^{\prime}-t^{\prime}\right\|^{2} \leq\left(\|\varphi\|^{2}+\|t\|^{2}+\left\|t^{\prime}\right\|^{2}\right)=C t e,
$$

where Cte is a positive constant. We then can extract a convergent subsequence

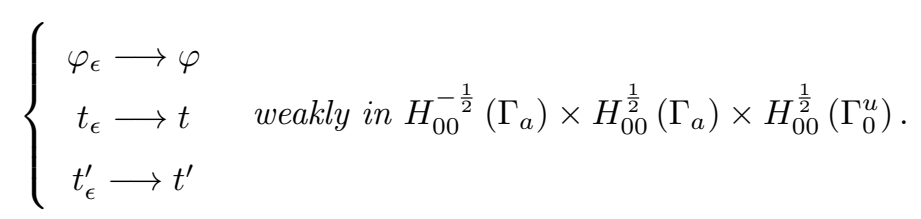

Since,

$$
0 \leq \lim _{\epsilon \longrightarrow 0}\left[\left\|\varphi_{\epsilon}-\varphi\right\|^{2}+\left\|t_{\epsilon}-t\right\|^{2}+\left\|t_{\epsilon}^{\prime}-t^{\prime}\right\|^{2}\right] \leq \lim _{\epsilon \rightarrow 0}\left[-\left\langle\varphi, \varphi_{\epsilon}-\varphi\right\rangle-\left\langle t, t_{\epsilon}-t\right\rangle-\left\langle t^{\prime}, t_{\epsilon}^{\prime}-t^{\prime}\right\rangle\right]=0
$$

we obtain that

$$
\left\{\begin{array}{l}
\varphi_{\epsilon} \longrightarrow \varphi \\
t_{\epsilon} \longrightarrow t \\
t_{\epsilon}^{\prime} \longrightarrow t^{\prime}
\end{array} \quad \text { strongly in } H_{00}^{-\frac{1}{2}}\left(\Gamma_{a}\right) \times H_{00}^{\frac{1}{2}}\left(\Gamma_{a}\right) \times H_{00}^{\frac{1}{2}}\left(\Gamma_{0}^{u}\right) .\right.
$$

Hence, $\exists h_{\epsilon} \geq 0 \underset{\epsilon \longrightarrow 0}{\longrightarrow}$, such that

$$
\left\|\varphi_{\epsilon}-\varphi\right\|+\left\|t_{\epsilon}-t\right\|+\left\|t_{\epsilon}^{\prime}-t^{\prime}\right\| \leq h_{\epsilon}
$$

Combining the inequalities (6.32) and (6.33), we obtain

$$
\left\|t_{\epsilon}^{\delta}-t_{\epsilon}\right\|+\left\|t_{\epsilon}^{\delta \prime}-t_{\epsilon}^{\prime}\right\|+\left\|t_{\epsilon}-t\right\|+\left\|t_{\epsilon}^{\prime}-t^{\prime}\right\| \leq \frac{\sqrt{2\left(k_{1}^{2}+k_{2}^{2}\right)}}{\epsilon}\|\delta\|+h_{\epsilon}
$$

The second estimation

According to the optimality conditions, we do: ((6.23) $-(5.20)) \times\left(t_{\epsilon}-t\right)+((6.24)-(5.21)) \times\left(\varphi_{\epsilon}-\varphi\right)+((6.23)$ $(5.20)) \times\left(t_{\epsilon}^{\prime}-t^{\prime}\right)$ and obtain

$$
\begin{aligned}
& \epsilon\left\langle t_{\epsilon}, t_{\epsilon}-t\right\rangle+\epsilon\left\langle t_{\epsilon}^{\prime}, t_{\epsilon}^{\prime}-t^{\prime}\right\rangle+\epsilon\left\langle\varphi_{\epsilon}, \varphi_{\epsilon}-\varphi\right\rangle+\left\|D\left(t_{\epsilon}-t\right)+E\left(t_{\epsilon}^{\prime}-t^{\prime}\right)\right\|^{2}+ \\
& +\left\|C\left(t_{\epsilon}-t\right)+B\left(\varphi_{\epsilon}-\varphi\right)\right\|^{2}+\left\|F\left(t_{\epsilon}^{\prime}-t^{\prime}\right)\right\|^{2}=0 .
\end{aligned}
$$

We have

$$
\begin{gathered}
\epsilon\left\langle t_{\epsilon}, t_{\epsilon}-t\right\rangle=\epsilon\left\|t_{\epsilon}-t\right\|^{2}+\epsilon\left\langle t, t_{\epsilon}-t\right\rangle, \\
\epsilon\left\langle t_{\epsilon}^{\prime}, t_{\epsilon}^{\prime}-t^{\prime}\right\rangle=\epsilon\left\|t_{\epsilon}^{\prime}-t^{\prime}\right\|^{2}+\epsilon\left\langle t^{\prime}, t_{\epsilon}^{\prime}-t^{\prime}\right\rangle, \\
\epsilon\left\langle\varphi_{\epsilon}, \varphi_{\epsilon}-\varphi\right\rangle=\epsilon\left\|\varphi_{\epsilon}-\varphi\right\|^{2}+\epsilon\left\langle\varphi, \varphi_{\epsilon}-\varphi\right\rangle .
\end{gathered}
$$

Replacingt by $C^{*} y_{1}+D^{*} y_{2}, t^{\prime}$ by $E^{*} y_{2}+F^{*} y_{3}$ and $\varphi$ by $B^{*} y_{1}$ respectively in the second terms of the right-hand side of equations (6.36), (6.37) and (6.38), respectively. Summing the three equations, we obtain

$$
\begin{aligned}
& \epsilon\left\langle t_{\epsilon}, t_{\epsilon}-t\right\rangle+\epsilon\left\langle t_{\epsilon}^{\prime}, t_{\epsilon}^{\prime}-t^{\prime}\right\rangle+\epsilon\left\langle\varphi_{\epsilon}, \varphi_{\epsilon}-\varphi\right\rangle=\epsilon\left\|t_{\epsilon}-t\right\|^{2}+\epsilon\left\langle\left(C^{*} y_{1}+D^{*} y_{2}\right),\left(t_{\epsilon}-t\right)\right\rangle \\
& +\epsilon\left\|t_{\epsilon}^{\prime}-t^{\prime}\right\|^{2}+\epsilon\left\langle\left(E^{*} y_{2}+F^{*} y_{3}\right),\left(t_{\epsilon}^{\prime}-t^{\prime}\right)\right\rangle+\epsilon\left\|\varphi_{\epsilon}-\varphi\right\|^{2}+\epsilon\left\langle B^{*} y_{1}, \varphi_{\epsilon}-\varphi\right\rangle .
\end{aligned}
$$


Regrouping the terms of $y_{1}, y_{2}$ and $y_{3}$ and applying the Young inequality we obtain

$$
\begin{aligned}
& \epsilon\left\langle t_{\epsilon}, t_{\epsilon}-t\right\rangle+\epsilon\left\langle t_{\epsilon}^{\prime}, t_{\epsilon}^{\prime}-t^{\prime}\right\rangle+\epsilon\left\langle\varphi_{\epsilon}, \varphi_{\epsilon}-\varphi\right\rangle \\
& \geq \epsilon\left\|t_{\epsilon}-t\right\|^{2}+\epsilon\left\|t_{\epsilon}^{\prime}-t^{\prime}\right\|^{2}+\epsilon\left\|\varphi_{\epsilon}-\varphi\right\|^{2}-\frac{\epsilon^{2}}{2}\left(\left\|y_{1}\right\|^{2}+\left\|y_{2}\right\|^{2}+\left\|y_{3}\right\|^{2}\right) \\
& -\frac{1}{2}\left(\left\|D\left(t_{\epsilon}-t\right)+E\left(t_{\epsilon}^{\prime}-t^{\prime}\right)\right\|^{2}+\left\|C\left(t_{\epsilon}-t\right)+B\left(\varphi_{\epsilon}-\varphi\right)\right\|^{2}+\left\|F\left(t_{\epsilon}^{\prime}-t^{\prime}\right)\right\|^{2}\right) .
\end{aligned}
$$

Injecting this last inequality in the left-hand side of equation (6.35), we obtain

$$
\epsilon\left\|t_{\epsilon}-t\right\|^{2}+\epsilon\left\|t_{\epsilon}^{\prime}-t^{\prime}\right\|^{2}+\epsilon\left\|\varphi_{\epsilon}-\varphi\right\|^{2} \leq \frac{\epsilon^{2}}{2}\left(\left\|y_{1}\right\|^{2}+\left\|y_{2}\right\|^{2}+\left\|y_{3}\right\|^{2}\right) .
$$

Hence, we have

$$
\left\|t_{\epsilon}-t\right\|+\left\|t_{\epsilon}^{\prime}-t^{\prime}\right\| \leq \sqrt{\epsilon} \sqrt{\left(\left\|y_{1}\right\|^{2}+\left\|y_{2}\right\|^{2}+\left\|y_{3}\right\|^{2}\right)} .
$$

We deduce the second estimation of Theorem 6.1

$$
\left\|t_{\epsilon}^{\delta}-t\right\|+\left\|t_{\epsilon}^{\prime \delta}-t^{\prime}\right\| \leq \frac{\|\delta\|}{\epsilon} \sqrt{2\left(k_{1}^{2}+k_{2}^{2}\right)}+\sqrt{\epsilon} \sqrt{\left\|y_{1}\right\|^{2}+\left\|y_{2}\right\|^{2}+\left\|y_{3}\right\|^{2}} .
$$

Finally, from (6.31) and (6.40), we also obtain

$$
\left\|t_{\epsilon}^{\delta}-t\right\|^{2} \leq \frac{\left(k_{1}^{2}+k_{2}^{2}\right)}{\epsilon^{2}}\|\delta\|_{L^{2}\left(\Gamma_{0}^{m}\right)}^{2}+\epsilon\left(\left\|y_{1}\right\|^{2}+\left\|y_{2}\right\|^{2}+\left\|y_{3}\right\|^{2}\right) .
$$

\subsection{Complete observation}

In this paragraph, we present an analysis of the Tikhonov regularization [24] in the cylindrical case with total observation of the potential on $\Gamma_{0}$. We will estimate the error between the exact and the regularized problem solution when the observation data $T$ is given on the whole boundary $\Gamma_{0}^{m}=\Gamma_{0}$, and evaluate the effect of noise when considering $(u=T+\delta)$ on $\Gamma_{0}$. We follow the approach developed by Morozov and Stessin [22]. We consider the following problem:

$$
\left(P_{0}\right)\left\{\begin{array}{ccc}
-\Delta u=0 & \text { in } & \Omega, \\
u=0 & \text { on } & \Sigma, \\
\frac{\partial u}{\partial n}=0 & \text { on } & \Gamma_{0}, \\
u=T & \text { on } & \Gamma_{0}, \\
u=t, \frac{\partial u}{\partial n}=\varphi & \text { on } & \Gamma_{a} .
\end{array}\right.
$$

Using the same definition of $u_{1}$ and $u_{2}$ as in $\left(\mathcal{P}_{1}, \mathcal{P}_{2}\right)$, and considering that $\Gamma_{0}^{u}=\emptyset$, the cost functional with total observation is:

$$
\begin{aligned}
J(\eta, \tau)= & \int_{\Omega}\left|\nabla u_{1}-\nabla u_{2}\right|^{2} d x d y . \\
J(\eta, \tau)= & C t e+(\eta, \tau) A\left(\begin{array}{l}
\eta \\
\tau
\end{array}\right)-2\langle\omega(T), P \tau\rangle . \\
& \inf _{\eta, \tau} J(\eta, \tau)=J(\varphi, t)=0 .
\end{aligned}
$$


where the operators matrix is defined by:

$$
A=\left(\begin{array}{cc}
Q & -Q P \\
-P Q & P
\end{array}\right) .
$$

For non negative values of $\epsilon$, the cost functional with the Tikhonov regularization is defined as follows

$$
\left.J_{\epsilon}(\eta, \tau)=C t e+(\eta, \tau) A\left(\begin{array}{l}
\eta \\
\tau
\end{array}\right)-2\langle\omega(T), P \tau\rangle+\epsilon\left(\|\eta\|_{\left(H_{00}^{\frac{1}{2}}\left(\Gamma_{a}\right)\right.}^{2}\right)^{\prime}+\|\tau\|_{H_{00}^{\frac{1}{2}}\left(\Gamma_{a}\right)}^{2}\right),
$$

We also define a cost function for the noisy data and Tikhonov regularization as follows

$$
\left.J_{\epsilon}^{\delta}(\eta, \tau)=C t e+(\eta, \tau) A\left(\begin{array}{l}
\eta \\
\tau
\end{array}\right)-2\langle\omega(T+\delta), P \tau\rangle+\epsilon\left(\|\eta\|_{\left(H_{00}^{\frac{1}{2}}\left(\Gamma_{a}\right)\right.}^{2}\right)^{\prime}+\|\tau\|_{H_{00}^{\frac{1}{2}}\left(\Gamma_{a}\right)}^{2}\right) .
$$

The aim of the following theorem is to give an estimate of the reconstructed electrical potential when considering noisy data on the complete accessible boundary. Following the same strategy used in the incomplete boundary case, we apply a Cholesky factorization of the operator $A$.

$$
A=R^{*} R \quad \text { where } R=\left(\begin{array}{cc}
B & C \\
0 & D
\end{array}\right)
$$

By identification, we have

$$
\left\{\begin{array}{l}
B=Q^{\frac{1}{2}}, \\
C=-Q^{\frac{1}{2}} P, \\
D=(P-P Q P)^{\frac{1}{2}},
\end{array}\right.
$$

One could check that operators $P, Q$ and $I-P Q$ are positive.

Theorem 6.3. Let's denote by $(\varphi, t)$, the exact solution and $\left(\varphi_{\epsilon}^{\delta, c}, t_{\epsilon}^{\delta, c}\right)$ the solution of the regularized problem with noisy data. Then, we have

$$
\left\|t_{\epsilon}^{\delta, c}-t\right\|_{H_{00}^{\frac{1}{2}\left(\Gamma_{a}\right)}} \leq \frac{k_{1}}{\epsilon}\|\delta\|_{L^{2}\left(\Gamma_{0}\right)}+g_{\epsilon}
$$

where

$$
g_{\epsilon} \geq 0 \text { and } g_{\epsilon} \longrightarrow 0 \text { for } \epsilon \longrightarrow 0
$$

Moreover, if $(\varphi, t) \in \operatorname{Im}\left(R^{*}\right)$ meaning that there exist $y_{1}, y_{2} \in L^{2}\left(\Gamma_{a}\right)$ such that $\varphi=B^{*} y_{1}$ and $t=C^{*} y_{1}+D^{*} y_{2}$, then we have

$$
\left\|t_{\epsilon}^{\delta, c}-t\right\|_{H_{00}^{\frac{1}{2}}\left(\Gamma_{a}\right)}^{2} \leq \frac{k_{1}^{2}}{\epsilon^{2}}\|\delta\|_{L^{2}\left(\Gamma_{0}\right)}^{2}+\epsilon\left(\left\|y_{1}\right\|^{2}+\left\|y_{2}\right\|^{2}\right)
$$

The proof of Theorem 6.3, uses exactly the same arguments developed in the proof of Theorem6.1. The second condition in the theorem is satisfied when the Cauchy data is sufficiently regular as shown in the following lemma.

Lemma 6.4. For a given electrical potential $T$ compatible with zero flux boundary condition, if $(t, \varphi)$ is solution of the data completion problem, then we can find $y_{1}$ and $y_{2}$ such as $y_{1} \in L^{2}(\theta), y_{2} \in L^{2}(\theta)$ such as $\varphi=B^{*} y_{1}$ and $t=C^{*} y_{1}+D^{*} y_{2}$, as soon as $T \in H^{\frac{3}{2}}(\theta)$.

The proof of the Lemma 6.4 is given in the appendix and it uses the modal decomposition of the Laplace operator on the transverse direction. 


\subsection{Theoretical interpretation of the measurement boundary size and the distribution of noise effects on the reconstructed solution}

In terms of convergence rate, the theoretical estimations shown in the Theorem 6.3 and Theorem 6.1 provide the same rate of convergence in terms of the noise $\delta$ and in terms of the regularization parameter $\epsilon$. The difference between complete and incomplete measurement cases is in the constants of the convergence. This could reflect if the one or the other is much more appropriate depending on the distribution of the noise. Let's denote by $E^{c}$ the maximal error on the electrical potential on the heart boundary $\Gamma_{a}$ in the case where we measure on the whole accessible $\Gamma_{0}$.

$$
\begin{aligned}
E^{c}(\epsilon, \delta): & =\frac{k_{1}^{2}}{\epsilon^{2}}\|\delta\|_{L^{2}\left(\Gamma_{0}\right)}^{2}+\epsilon\left(\left\|y_{1}\right\|^{2}+\left\|y_{2}\right\|^{2}\right) \\
& =\frac{k_{1}^{2}}{\epsilon^{2}}\left(\|\delta\|_{L^{2}\left(\Gamma_{0}^{m}\right)}^{2}+\|\delta\|_{L^{2}\left(\Gamma_{0}^{u}\right)}^{2}\right)+\epsilon\left(\left\|y_{1}\right\|^{2}+\left\|y_{2}\right\|^{2}\right)
\end{aligned}
$$

On the other side, we denote by $E^{i}$ the maximal error in the case where we measure only on the subboundary $\Gamma_{0}^{m}$

$$
E^{i}\left(\Gamma_{0}^{m}, \epsilon, \delta\right):=\frac{\left(k_{1}^{2}+k_{2}^{2}\right)}{\epsilon^{2}}\|\delta\|_{L^{2}\left(\Gamma_{0}^{m}\right)}^{2}+\epsilon\left(\left\|y_{1}\right\|^{2}+\left\|y_{2}\right\|^{2}+\left\|y_{3}\right\|^{2}\right)
$$

The difference between both error bounds reads

$$
E^{c}(\epsilon, \delta)-E^{i}\left(\Gamma_{0}^{m}, \epsilon, \delta\right)=\frac{k_{1}^{2}}{\epsilon^{2}}\|\delta\|_{L^{2}\left(\Gamma_{0}^{u}\right)}^{2}-\frac{k_{2}^{2}}{\epsilon^{2}}\|\delta\|_{L^{2}\left(\Gamma_{0}^{m}\right)}^{2}-\epsilon\left\|y_{3}\right\|^{2}
$$

From this we distinguish two cases depending on the distribution of the noise $\delta$ :

- If $\|\delta\|_{L^{2}\left(\Gamma_{0}^{u}\right)}^{2}<\frac{k_{2}^{2}}{k_{1}^{2}}\|\delta\|_{L^{2}\left(\Gamma_{0}^{m}\right)}^{2}+\frac{\epsilon^{3}}{k_{1}^{2}}\left\|y_{3}\right\|$, then it is worth to use all the available measurement at $\Gamma_{0}$.

- If $\|\delta\|_{L^{2}\left(\Gamma_{0}^{u}\right)}^{2}>\frac{k_{2}^{2}}{k_{1}^{2}}\|\delta\|_{L^{2}\left(\Gamma_{0}^{m}\right)}^{2}+\frac{\epsilon^{3}}{k_{1}^{2}}\left\|y_{3}\right\|$, then it is worth to only use the measurements on $\Gamma_{0}^{m}$

Remark 6.5. In practice $\left\|y_{3}\right\|$ is not available, but since this term is multiplied by $\epsilon^{3}$, the term $\frac{\epsilon^{3}}{k_{1}^{2}}\left\|y_{3}\right\|$ might be neglected. This problem could be avoided using simulated data for which one has exact solution and thus could construct $y_{1}, y_{2}, y_{3}$. Then one could test if the distribution of noise is much more suitable for full observation on $\Gamma_{0}$ or incomplete mesaurements on $\Gamma_{0}^{m}$ using simulated data.

Remark 6.6. When interpolation is performed like in [11,13,23] it is important to build an estimator of the interpolation error, which could then be used to decide whether or not to use the interpolated data.

Remark 6.7. The error bounds here do not take into account the errors related to the discretization of the problem. It would be interesting to add the discretization error to the comparison result.

\section{NUMERICAL METHOD}

In this section, we present the results of numerical simulations to solve the data completion problem $\left(p_{0}\right)$ on a rectangular area using the invariant embedding technique developed in the previous sections. The idea is to test the effect and the area that lacks data method for a problem where an analytical solution is known. Thus it is possible to accurately compare results that are "reconstructed" and "analytical".

We consider problem $\left(p_{0}\right)$ on $] 0, a[\times] 0,2 \pi[. \quad x$ is the variable which describes the area of length between 0 and $a$, while $y$ is the one which crosses the transverse coordinate between 0 and $\pi$. The Cauchy data on $\Gamma_{0}$ are $u(x, y)_{\mid \Gamma_{0}^{m}}=u(0, y)_{\mid \Gamma_{0}^{m}}=T=\sin (y)$ and $\frac{\partial u}{\partial n} u(x, y)_{\mid \Gamma_{0}}=\frac{\partial u}{\partial n}(0, y)_{\mid \Gamma_{0}}=0$ and is seeking to rebuild the data on the surface $\Gamma_{a}$. One could check that the harmonic function $u(x, y)=\cosh (x) \sin (y)$ is the solution of the problem. The data to be completed on $\Gamma_{a}$ are: $t_{\text {theo }}=u(a, y)=\cosh (a) \sin (y)$ and 
$\varphi_{\text {theo }}=\frac{\partial u}{\partial n}(a, y)=\sinh (a) \sin (y)$ and on $\Gamma_{0}^{u}$ we also construct $t_{\text {theo }}^{\prime}=u(0, y)=\sin (y)$. We use a finite difference method for the numerical solution of (4.5) and (4.7). To do this we denote by $n$ the number of points along the length $x$-direction and $p$ the number of points along the transverse direction $y$. We define by $u(i h, j k)$ the solution of the problem at the grid points coordinates $x=i h, y=j k$. This discretization is done in two separate steps. The first follows the transverse direction and that turns the discrete Dirichlet-Neumann and Neumann-Dirichlet operators in matrices respectively $P_{i}$ and $Q_{i}$ at $x=i h, 0 \leq i \leq n$. These matrices are then of size $(p-1) \times(p-1)$ that connects the values $u_{i}$ of the solution at the grid points on section $x=i h$ to the approximation of derivative on the same section. These matrices satisfy Riccati equations along the $x$ variable. The second step consists in the discretization along the axis of dipping, that is to say in $x$. It then applies a Euler scheme operators to resolve and determine on the surface $x=a$ is the same procedure for solving the residuals. The first discretization defines the matrix of size $\omega(p-1) \times 1$ and also checking the equations residue. The second allows, also using an Euler scheme to determine the $\omega$ on the surface for missing data. Finally, we must build the interface operator and solve linear problems to determine $t_{\text {num }}$ and $t_{\text {num }}^{\prime}$. After a discretization in the $x$ direction, this equation is solved by an explicit Euler scheme respecting stability conditions.

$$
\left\{\begin{array}{c}
P_{i}=P_{i-1}\left(I-h P_{i-1}\right)-h \Delta_{T, k}, \\
P(0)_{\mid \Gamma_{0}}=0 .
\end{array}\right.
$$

where $\Delta_{T, k}$ is the three diagonal matrix associated with the three points approximation to the second derivative. A similar scheme is applied to the Neumann-Dirichlet operator

$$
\left\{\begin{array}{c}
Q_{i}=Q_{i-1}\left(I-h \Delta_{T, k} Q_{i-1}\right)-h I, \\
Q(0)_{\mid \Gamma_{0}^{m}}=0, \quad Q(0)_{\mid \Gamma_{0}^{u}}=0 .
\end{array}\right.
$$

and to the residual equation :

$$
\left\{\begin{array}{l}
\omega_{i}=\left(I-h \Delta_{T, k} Q_{i-1}\right) \omega_{i-1} \\
\omega(0)_{\mid \Gamma_{0}^{m}}=0, \quad \omega(0)_{\mid \Gamma_{0}^{u}}=0
\end{array},\right.
$$

The equations of operators $S P^{\prime}$ and $H$ are solved similarly. The scheme has been implemented in MATLAB. In order to be able to compare the results with different level of noise, we generated a normalized white noise using the rand function of matlab. This noise is saved and is used for all test cases, it will be just fully or partially rescaled in order to produce different levels of noise. In the following simulations we took $p=201$, $n=2000, a=\pi b=2 \pi$ and $\epsilon=10^{-6}$. We shall also say that in what follows the measurement boundary is placed in the center of $\Gamma_{0}$. For a given percentage $\alpha$, we define $\Gamma_{0}^{m}$ as the interval $\left[\frac{b}{2}\left(1-\frac{\alpha}{100}\right), \frac{b}{2}\left(1+\frac{\alpha}{100}\right)\right]$

\section{REsults}

In this section we will conduct to two test cases reflecting the theoretical results that we obtained. In the first test case we will use a homogeneous white noise, which means that the level of noise is the same in all the boundary $\Gamma_{0}$. This does not mean that the noise is constant, it only means that point-wise, the absolute value of the noise is less than a given constant. In this case, we are mimicking the fact that the electrodes cover the whole accessible domain $\Gamma_{0}$ and that the electrodes have the same measurement error. We will look how loosing part of these electrodes measurements affects the reconstructed solution.

In the second case, we consider that either part of electrodes are missing or that it is measuring but with a high level of noise. In the case that part of the electrodes is missing we suppose that one could use an interpolation method and depending on the error of interpolation we will look if considering these interpolated signals improves or deteriorates the solution. 


\subsection{Homogeneous noise}

In this paragraph, we test the effect of size of the sub-boundary of measurements $\Gamma_{0}^{m}$ and the different levels of noise on the quality of the reconstructed solution on the inaccessible boundary $\Gamma_{a}$. We vary the size of $\Gamma_{0}^{m}$ from $20 \%$ to $100 \%$ of the full accessible boundary. We also vary the level of noise from $0 \%$ to $30 \%$. In figure 4 (respectively, figure 4), we measure $T$ on $50 \%$ (respectively, $90 \%$ ) of $\Gamma_{0}$, the measurements contain $10 \%$ (respectively, 20\%) of noise as shonw in the left panel. The obtained solution on the "heart" boundary $\Gamma_{a}$ is shown in the left panel (red continuous line) where we compare it to the exact solution (blue dashed line). The relative error with respect to the exact solution is 0.26 (respectively, 0.18 ).
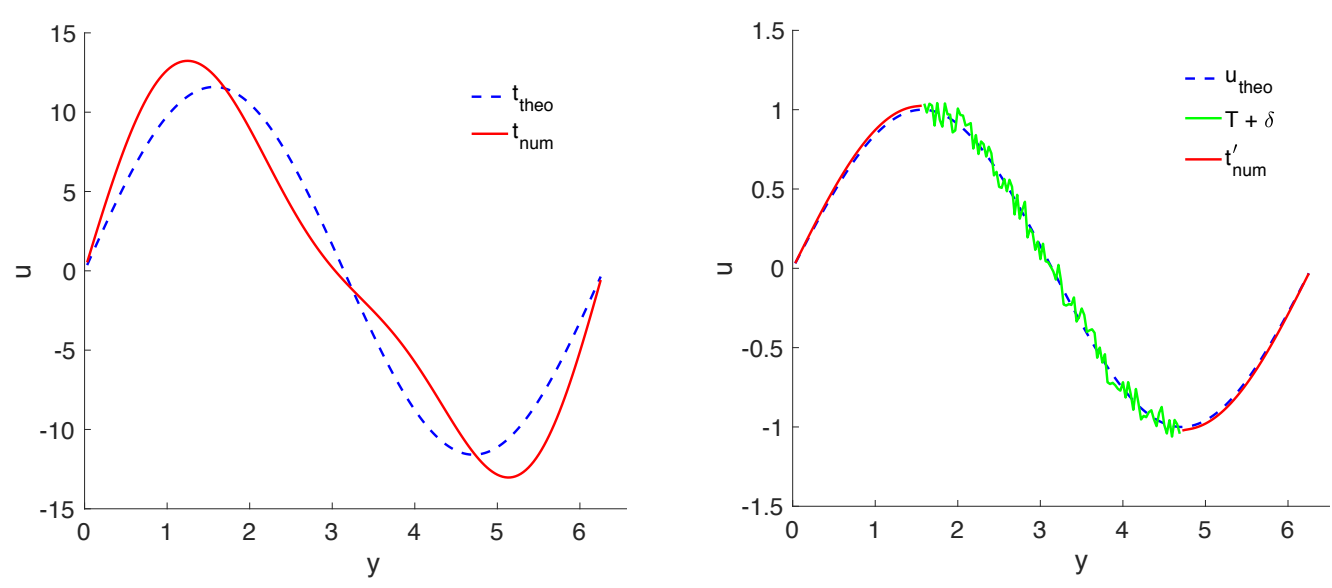

Figure 3. Left: Comparison between the exact solution (blue dashed ligne) and the incomplete boundary measurement inverse solution (red continuous line). Right solutions with respect to the noise level at $\Gamma_{0}^{u}$. The noise on the measured boundary $\Gamma_{0}^{m}$ does not not change $\Gamma_{0}^{m}$ occupies $50 \%$ of $\Gamma_{0}$ and the noise level is $10 \%$. Right: Reconstructed solution on the accessible unmeasured boundary $\Gamma_{0}^{u}$ (red continuous line), Noisy measurement $T+\delta$ (green continuous line) and exact solution on $\Gamma_{0}$ (blue dashed line).

In Table 8.1, we present the relative error of the reconstructed potential $t_{\text {num }}$ on $\Gamma_{a}$ with respect to the theoretical solution. Looking at each raw of the Table 8.1, we remark that the relative error increases monotonously when increasing the level of noise and this is independent of size of the measurement domain $\Gamma_{0}^{m}$. On the other side, looking at the columns of Table 8.1, we remark that the error does not decrease monotonously when increasing the size of the measurement boundary $\Gamma_{0}^{m}$ for each level of noise. Although, it is the case for low level of noise $(0 \%, 1 \%, 5 \%, 10 \%)$, for high level of noise $(20 \%, 30 \%)$, we see the error increases when the size of $\Gamma_{0}^{m}$ increases from $20 \%$ to $30 \%$, and then decreases monotonously. We think that the distribution of the noise is the main reason.

\subsection{Non homogeneous noise}

Here we suppose that either some electrodes are defective or certain regions of the accessible boundary are not covered by electrodes. We also suppose that the user want to make some interpolation on this region. We assume here that the error of interpolation would be combined to the error of measurement leading to a new noise level on the boundary $\Gamma_{0}^{u}$ different from the noise on $\Gamma_{0}^{m}$. In what follows we don't do any interpolation we progressively increase the level of noise on the unmeasured boundary $\Gamma_{0}^{u}$ and we compare the solutions of the following two cases: In the first case, we only use the measurements recorded by the non defective electrodes on $\Gamma_{0}^{m}$ and solve the inverse problem with an incomplete information on the accessible boundary we denote the relative error $E^{i}$ as previously denoted in the analysis section. In the second case we use the 

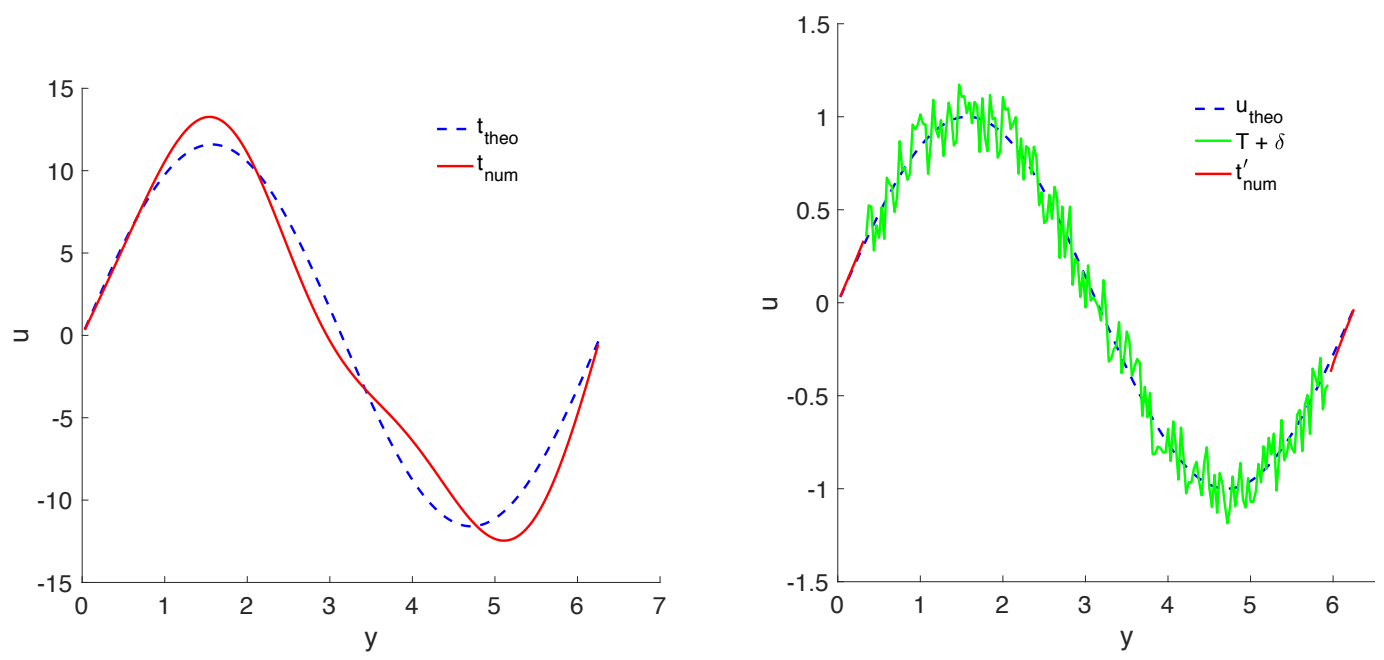

Figure 4. Left: Comparison between the exact solution (blue dashed ligne) and the incomplete boundary measurement inverse solution (red continuous line). Right solutions with respect to the noise level at $\Gamma_{0}^{u}$. The noise on the measured boundary $\Gamma_{0}^{m}$ does not not change $\Gamma_{0}^{m}$ occupies $50 \%$ of $\Gamma_{0}$ and the noise level is $10 \%$. Right: Reconstructed solution on the accessible unmeasured boundary $\Gamma_{0}^{u}$ (red continuous line), Noisy measurement $T+\delta$ (green continuous line) and exact solution on $\Gamma_{0}$ (blue dashed line).

\begin{tabular}{|c|c|c|c|c|c|c|}
\hline \multicolumn{1}{|c|}{ noise $\%$} & $0 \%$ & $1 \%$ & $5 \%$ & $10 \%$ & $20 \%$ & $30 \%$ \\
\hline$\Gamma_{0}^{m \%}$ & 0.6151 & 0.6169 & 0.6261 & 0.6417 & 0.6847 & 0.7414 \\
$30 \%$ & 0.4358 & 0.4491 & 0.5078 & 0.5901 & 0.7723 & 0.9666 \\
$50 \%$ & 0.0894 & 0.1067 & 0.1769 & 0.2655 & 0.4433 & 0.6213 \\
$60 \%$ & 0.0312 & 0.0394 & 0.1076 & 0.2036 & 0.3989 & 0.5949 \\
$80 \%$ & 0.0062 & 0.0166 & 0.0710 & 0.1399 & 0.2780 & 0.4160 \\
$90 \%$ & 0.0059 & 0.0116 & 0.0480 & 0.0950 & 0.1891 & 0.2833 \\
$100 \%$ & 0.0059 & 0.0084 & 0.0275 & 0.0532 & 0.1052 & 0.1572 \\
\hline
\end{tabular}

TABLE 1. Relative error between the exact solution and the solution obtained using different measurement subboundaries (raws) and different level of noise (columns), $(a=\pi, b=2 \pi)$.

noisy data in all the accessible boundary $\Gamma_{0}$ and we denote by $E^{c}$ the corresponding relative error. In Figure 5 (left, respectively right) the measurement boundary $\Gamma_{0}^{m}$ occupies $80 \%$ (respectively, 50\%) of $\Gamma_{0}$. Using a noise level of $2 \%$ (respectively, $5 \%$ ), the relative error $E^{i}$ is 0.03 (respectively, 0.17 ) see blue constant line in both panels. We remark that for a noise level less than $14 \%$ (respectively $30 \%$ ) on $\Gamma_{0}^{u}$, while keeping the noise level $2 \%$ (respectively $5 \%$ ) on $\Gamma_{0}^{m}$, the relative error of the complete measurements case solution is better than incomplete case solution, the red line is under the blue line. While for higher level of noise on $\Gamma_{0}^{m}$, the complete measurement case solution becomes worst than the incomplete one. This reflects the theoretical result that we obtained in the section of comparison between $E^{i}$ and $E^{c}$. 

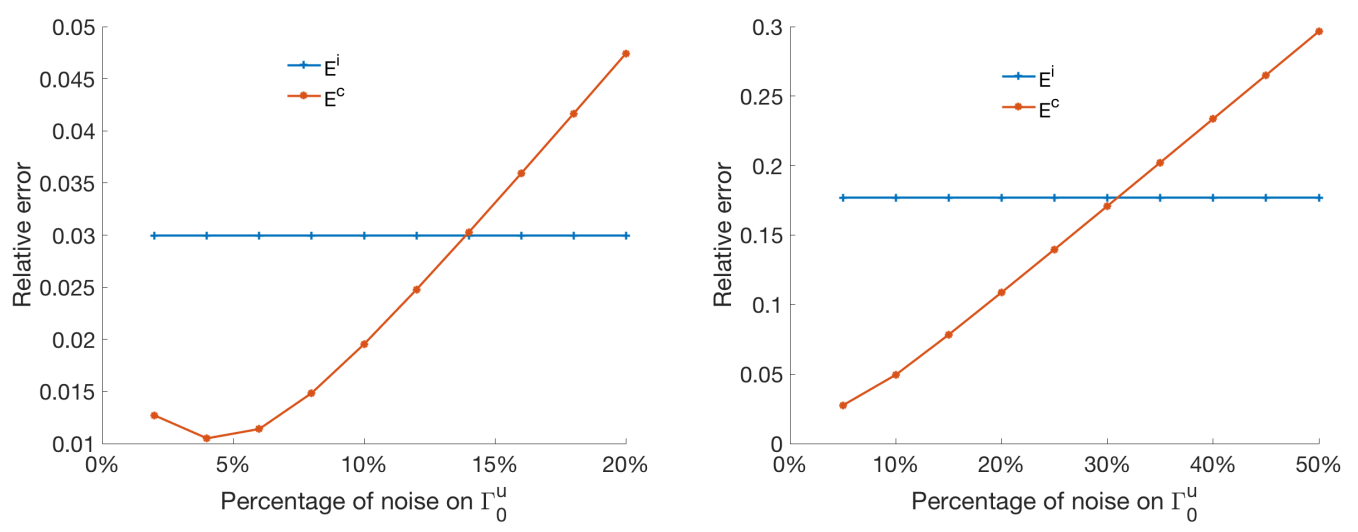

Figure 5. Comparison between the incomplete boundary measurment (blue ligne) and the complete boundary measurement (red line) inverse solutions with respect to the noise level at $\Gamma_{0}^{u}$. The noise on the measured boundary $\Gamma_{0}^{m}$ does not not change. Left figure $\Gamma_{0}^{m}$ occupies $80 \%$ and the fixed noise is $2 \%$. Right figure $\Gamma_{0}^{m}$ occupies $50 \%$ and the fixed noise is $5 \%$.

\subsection{An Oscillating case}

In order to show how the method performs in an oscillating case, we change here the analytical expression of the solution by adding another frequency in the y-direction. The new function we look for is

$$
u(x, y)=\sin (y) \cosh (x)+4 \sin \left(\frac{y}{2}\right) \cosh \left(\frac{x}{2}\right)
$$

the space domain is $[0,1.5 \pi] \times[0,8 \pi]$. The measured boundary is $\Gamma_{0}^{m}=0 \times[0.8 \pi, 7.2 \pi]$ which corresponds to $80 \%$ of $\Gamma_{0}$ and the noise percentage $\delta=20 \%$ see Figure 6 (right). The relative error of the solution shown in Figure 6 (left) is $13 \%$.

\section{CONCLUSION}

In this paper, we presented a method to solve a data completion problem for the electrocardiography imaging inverse problem. The Cauchy data are measured on the part of the accessible boundary. In our case the Neumann boundary condition is given in the whole accessible domain. We used a method based on the factorization of elliptic boundary value problems. We analyzed the error in case of measurements on all the accessible domain and when only part of the accessible domain is measured. We obtained a comparison result between both cases depending on the distribution of the noise on $\Gamma_{0}$ We numerically tested this method on a $2 \mathrm{D}$ rectangular domain. We obtained the following results: 1) for a fixed $\Gamma_{0}^{m}$, the error increases monotonously with the level of noise and this is independent of size of the measurement domain $\Gamma_{0}^{m} ; 2$ ) for a fixed noise level, there is no guarantee that error decreases while increasing the size of the measurement boundary $\Gamma_{0}^{m}$. Our conclusion is that depends on how the noise is distributed in $\Gamma_{0} .3$ ) by progressively increasing the noise level on $\Gamma_{0}^{u}$ while the noise level is fixed on $\Gamma_{0}^{m}$, one could numerically find the critical noise level on $\Gamma_{0}^{u}$ from which there is no gain in considering the data $\Gamma_{0}^{u}$. This result could be practically used if one has an estimator of the interpolation error in the unmeasured boundary. The numerical analysis of this problem taking into account 3D realistic data both in the geometry and the measurements, taking into account the noise in the measured boundary but also the numerical errors in the computation of all the operators would be subject of a forthcoming work. 

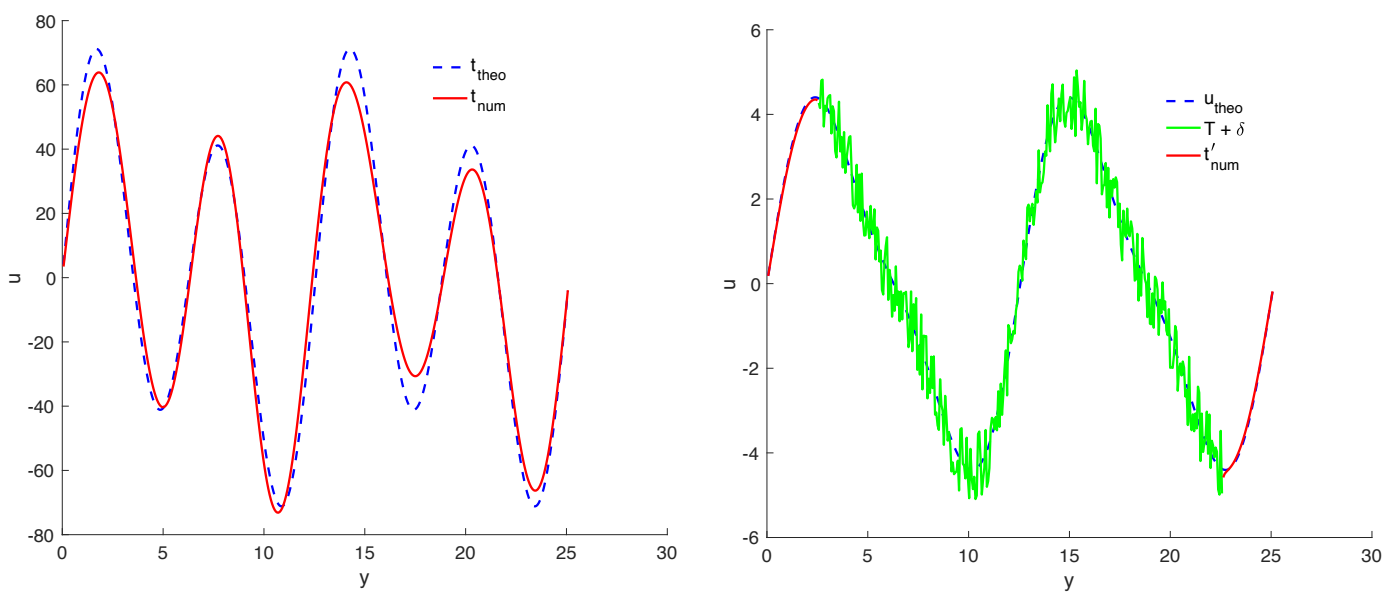

Figure 6. Comparison between the exact solution (blue dashed ligne) and the incomplete boundary measurement inverse solution (red continuous line). Right solutions with respect to the noise level at $\Gamma_{0}^{u}$. The noise on the measured boundary $\Gamma_{0}^{m}$ does not not change $\Gamma_{0}^{m}$ occupies $80 \%$ of $\Gamma_{0}$ and the noise level is $20 \%$ (left). Reconstructed solution on the accessible unmeasured boundary $\Gamma_{0}^{u}$ (red continuous line), Noisy measurement $T+\delta$ (green continuous line) and exact solution on $\Gamma_{0}$ (blue dashed line) (right).

\section{ACKNOWLEDGements.}

This work has been supported by EPICARD cooperative research program, funded by INRIA international laboratory LIRIMA. The LAMSIN researcher's work is supported on a regular basis by the Tunisian Ministry of Higher Education, Scientific Research and Technology. This study received also financial support from the French Government as part of the 'Investments of the future' program managed by the National Research Agency (ANR), Grant reference ANR-10-IAHU-04.

\section{Appendix: proof of LEMma 6.4}

Proof 9.1. Since we look for $y_{1}$ and $y_{2}$ in $L^{2}(\theta)$ such that $\varphi=B^{*} y_{1}$ and $t=C^{*} y_{1}+D^{*} y_{2}$, we formally have $\varphi=Q^{\frac{1}{2}} y_{1}$. This means that

$$
y_{1}=Q^{-\frac{1}{2}} \varphi .
$$

On the other side, we have $\varphi=P t$, this means that $t=P^{-1} \varphi$

$$
t=P^{-1} \varphi=D^{*} y_{2}-C^{*} y_{1}=D^{*} y_{2}-P \varphi .
$$

This means that $D^{*} y_{2}=\left(P^{-1}+P\right) \varphi$.

We begin by proving that we can find $y_{2}$ such that $D^{*} y_{2}=\left(P^{-1}+P\right) \varphi$. We decompose the Dirichlet data $T$ on the basis of eigenfunctions $\left(e_{i}\right)_{i>0}$ of $\Delta_{\theta}$ the Laplacian on the transverse direction $\theta$;

$$
T=\sum_{i} T_{i} e_{i}
$$

Then we have

$$
t=\sum_{i} T_{i} \exp \left(-\int_{0}^{a} \lambda_{i} \tanh \left(\lambda_{i} x\right) d x\right) e_{i}
$$


and since $\varphi=P t$, we have

$$
\varphi=\sum_{i} \lambda_{i} T_{i} \tanh \left(\lambda_{i} a\right) \exp \left(-\int_{0}^{a} \lambda_{i} \tanh \left(\lambda_{i} x\right) d x\right) e_{i}
$$

we multiply by $\left(P^{-1}+P\right)$ :

$$
\left(P^{-1}+P\right) \varphi=\sum_{i}\left(\lambda_{i}^{2} \tanh ^{2}\left(\lambda_{i} a\right)+1\right) T_{i} \exp \left(-\int_{0}^{a} \lambda_{i} \tanh \left(\lambda_{i} x\right) d x\right) e_{i}
$$

we remark that

$$
\exp \left(-\int_{0}^{a} \lambda_{i} \tanh \left(\lambda_{i} x\right) d x\right)=\frac{1}{\cosh \left(\lambda_{i} a\right)} .
$$

On the orther side, we have $D=(P-P Q P)^{\frac{1}{2}}$, its eigenvalues are

$$
\left(\lambda_{i} \tanh \left(\lambda_{i} a\right)\right)^{\frac{1}{2}} \frac{1}{\cosh \left(\lambda_{i} a\right)} .
$$

Thus we can define $y_{2}$ by

$$
\begin{aligned}
y_{2} & =\sum_{i} \frac{\left(\lambda_{i}^{2} \tanh ^{2}\left(\lambda_{i} a\right)+1\right)}{\cosh \left(\lambda_{i} a\right)} \cdot \frac{\cosh \left(\lambda_{i} a\right)}{\left(\lambda_{i} \tanh \left(\lambda_{i} a\right)\right)^{\frac{1}{2}}} T_{i} e_{i} \\
& =\sum_{i} \frac{1}{\left(\lambda_{i} \tanh \left(\lambda_{i} a\right)\right)^{\frac{1}{2}}}+\left(\lambda_{i} \tanh \left(\lambda_{i} a\right)\right)^{\frac{3}{2}} T_{i} e_{i}
\end{aligned}
$$

Hence, $y_{2}$ is well defined in $L^{2}(\theta)\left(\sum_{i}\left(y_{2}^{i}\right)^{2}<+\infty\right)$ as soon as $\sum_{i} \lambda_{i}^{3} T_{i}^{2}<+\infty$, which is equivalent to the fact that $T \in H^{\frac{3}{2}}(\theta)$.

Using the same strategy, we can find $y_{1}$ defined as follows

$$
\begin{aligned}
y_{1} & =Q^{-\frac{1}{2}} \varphi \\
& =\sum_{i} \frac{\left(\lambda_{i}\right)^{\frac{1}{2}}}{\left(\tanh \left(\lambda_{i} a\right)\right)^{\frac{1}{2}}} \lambda_{i} \frac{\tanh \left(\lambda_{i} a\right)}{\cosh \left(\lambda_{i} a\right)} T_{i} e_{i} \\
& =\sum_{i}\left(\lambda_{i}\right)^{\frac{3}{2}} \frac{\left(\tanh \left(\lambda_{i} a\right)\right)^{\frac{1}{2}}}{\cosh \left(\lambda_{i} a\right)} T_{i}^{\prime} e_{i} .
\end{aligned}
$$

We have $y_{1} \in L^{2}\left(\sum_{i}\left(y_{1}^{i}\right)^{2}<+\infty\right)$ for $T \in H^{\frac{3}{2}}(\theta)$.

\section{REFERENCES}

[1] Amel Ben Abda, Jacques Henry, and Fadhel Jday. Boundary data completion: the method of boundary value problem factorization. Inverse Problems, 27(5):055014, 2011.

[2] Rajae Aboulaich, Amel Ben Abda, and Moez Kallel. Missing boundary data reconstruction via an approximate optimal control. Inverse Problems \& Imaging, 2(4):411-426, 2008. 
[3] Rajae Aboulaich, Amel Ben Abda, and Moez Kallel. A control type method for solving the cauchy-stokes problem. Applied mathematical modelling, 37(6):4295-4304, 2013.

[4] Rajae Aboulaich, Najib Fikal, E El Guarmah, and N Zemzemi. Stochastic finite element method for torso conductivity uncertainties quantification in electrocardiography inverse problem. Mathematical Modelling of Natural Phenomena, 11(2):119, 2016.

[5] Stephane Andrieux, Amel Ben Abda, and Thouraya Nouri Baranger. Data completion via an energy error functional. Comptes Rendus Mécanique, 333(2):171-177, 2005.

[6] Mejdi Azaïez, Faker Ben Belgacem, and Henda El Fekih. On cauchy's problem: Ii. completion, regularization and approximation. Inverse problems, 22(4):1307, 2006.

[7] Roger C Barr, Maynard Ramsey III, and Madison S Spach. Relating epicardial to body surface potential distributions by means of transfer coefficients based on geometry measurements. Biomedical Engineering, IEEE Transactions on, pages 1-11, 1977.

[8] Laura Bear, Mark Potse, Josselin Duchateau, Nejib Zemzemi, Yves Coudière, and Rémi Dubois. Accuracy of lead removal vs linear interpolation in non-invasive electrocardiographic imaging (ecgi). In Computing in Cardiology Conference (CinC), 2015. IEEE, 2014.

[9] Richard Ernest Bellman and Howard A Osborn. Dynamic programming and the variation of Green's functions. RAND Corporation, 1957.

[10] Julien Bouyssier, Nejib Zemzemi, and Jacques Henry. Inverse problem in electrocardiography via factorization method of boundary problems: how reconstruct epicardial potential maps from measurements of the torso? In International Symposium on Biomedical Imaging (ISBI), 2015. IEEE, 2015.

[11] John E Burnes, David C Kaelber, Bruno Taccardi, Robert L Lux, Philip R Ershler, and Yoram Rudy. A field-compatible method for interpolating biopotentials. Annals of biomedical engineering, 26(1):37-47, 1998.

[12] A Chakib and A Nachaoui. Convergence analysis for finite element approximation to an inverse cauchy problem. Inverse Problems, 22(4):1191, 2006.

[13] Alireza Ghodrati, Dana H Brooks, and Robert S MacLeod. Methods of solving reduced lead systems for inverse electrocardiography. Biomedical Engineering, IEEE Transactions on, 54(2):339-343, 2007.

[14] Jacques Hadamard. Lectures on Cauchy's problem in linear partial differential equations. Courier Corporation, 2014.

[15] Nejla Hariga-Tlatli, TN Baranger, and Jocelyne Erhel. Misfit functional for recovering data in 2d electrocardiography problems. Engineering Analysis with Boundary Elements, 34(5):492-500, 2010.

[16] Jacques Henry and AM Ramos. Factorization of second-order elliptic boundary value problems by dynamic programming. Nonlinear Analysis: Theory, Methods $\& 3$ Applications, 59(5):629-647, 2004.

[17] Jacques Henry and Angel M Ramos. Study of the initial value problems appearing in a method of factorization of second-order elliptic boundary value problems. Nonlinear Analysis: Theory, Methods 63 Applications, 68(10):2984-3008, 2008.

[18] Jacques Henry and Angel M Ramos. La méthode de factorisation des problèmes aux limites par plongement invariant. ISTE editions, 2016.

[19] Fadhel Jday. Complétion de données frontieres: la méthode de plongement invariant. PhD thesis, PHD thesis, 2012.

[20] Vladimir Arkad'evich Kozlov, Vladimir Gilelevich Maz'ya, and AV Fomin. An iterative method for solving the cauchy problem for elliptic equations. Zhurnal Vychislitel'noi Matematiki i Matematicheskoi Fiziki, 31(1):64-74, 1991.

[21] Jacques Louis Lions and Enrico Magenes. Problemes aux limites non homogenes et applications. Dunod, 1968.

[22] Vladimir Alekseevich Morozov and Michael Stessin. Regularization methods for ill-posed problems. CRC press Boca Raton, FL: 1993.

[23] Thom F Oostendorp, Adriaan van Oosterom, and Geertjan Huiskamp. Interpolation on a triangulated 3d surface. Journal of Computational Physics, 80(2):331-343, 1989.

[24] A.N. Tikhonov and V. Arsenin. Solutions of ill-posed problems. WH Winston, Washington, DC, 1977.

[25] Yong Wang and Yoram Rudy. Application of the method of fundamental solutions to potential-based inverse electrocardiography. Annals of biomedical engineering, 34(8):1272-1288, 2006.

[26] Nejib Zemzemi. A steklov-poincaré variational formulation of the inverse problem in cardiac electrophysiology. In CinCComputing in Cardiology Conference, page 193. IEEE, 2013.

[27] Nejib Zemzemi, Hamed Bourenane, and Hubert Cochet. An iterative method for solving the inverse problem in electrocardiography imaging: From body surface to heart potential. In Computing in Cardiology Conference (CinC), 2014, pages 717-720. IEEE, 2014. 